\title{
Dynamics of a delayed phytoplankton-zooplankton system with Crowley-Martin functional response
}

Tiancai Liao ${ }^{1,2}$, Hengguo $\mathrm{Yu}^{1,2}$ and Min Zhao $2,3^{*}$

"Correspondence: zmcn@tom.com ${ }^{2}$ School of Life and Environmental Science, Wenzhou University, Wenzhou, Zhejiang 325035, China ${ }^{3}$ Zhejiang Provincial Key Laboratory for Water Environment and Marine Biological Resources Protection, Wenzhou University, Wenzhou, Zhejiang 325035, China Full list of author information is available at the end of the article

\section{Springer}

\begin{abstract}
In this paper, a delayed phytoplankton-zooplankton system with Crowley-Martin functional response is investigated analytically. We study the permanence and analyze the stability of the both boundary and positive equilibrium points for the system with delay as well as the system without delay. The global asymptotic stability is discussed by constructing a suitable Lyapunov functional. Numerical analysis indicates that the delay does not change the stability of the positive equilibrium point. Furthermore, we also show that due to the increase of the delay there occurs a Hopf bifurcation of periodic solutions. It is found that population fluctuations will not appear under the condition of certain parameters. In addition, we determine the direction of the Hopf bifurcation and the stability of bifurcating periodic solutions by applying a normal form method and center manifold theory. Finally, some numerical simulations are carried out to support our theoretical analysis results.
\end{abstract}

Keywords: stability; delay; Hopf bifurcation; Lyapunov functional; periodic solution

\section{Introduction}

Plankton plays an important role in the ocean and the climate because of their participation in the global carbon cycle at the base of the food chain [1]. There are two forms of plankton, the plant forms of the plankton community are known as phytoplankton and the animals in the plankton community are known as zooplankton. Phytoplankton biomass has the characteristics of rapid proliferation, this change is called bloom [2]. Under some circumstances, however, phytoplankton bloom can appear and affect the ecological balance, and it even can endanger human life and health. Therefore, a better understanding of mechanisms that determine the plankton dynamics is of considerable interest [3].

In recent years, there were many experimental ecologists as well as mathematical ecologists who have paid more attention to the phenomena of phytoplankton blooms. And many scholars proposed different deterministic models to study the dynamical behavior of the plankton system and tried to explain the dynamic mechanism of phytoplankton in different ways [4-11]. Abbas et al. [12] considered the two species competitive delay plankton allelopathy stimulatory deterministic model and investigated the existence and uniqueness of the solution as well as the persistence and the stability properties of the model, which is very useful and meaningful for the study of plankton bloom. Chakraborty and

(c) The Author(s) 2017. This article is distributed under the terms of the Creative Commons Attribution 4.0 International License (http://creativecommons.org/licenses/by/4.0/), which permits unrestricted use, distribution, and reproduction in any medium, provided you give appropriate credit to the original author(s) and the source, provide a link to the Creative Commons license, and indicate if changes were made. 
Das [13] investigated some properties of a two-zooplankton one-phytoplankton system that exhibits a Holling type II functional response in the presence of toxicity, the results they obtained can provide great help for researching the dynamic complexity of plankton systems. Kartal et al. [14] proposed a phytoplankton-zooplankton system via a new approach by using a system of differential equations with piecewise constant arguments and studied the biological dynamics of the bloom in the plankton system. It is significant that they explained the plankton bloom depended on three different parameters, namely $\theta$ (rate of toxin production per phytoplankton), $\beta$ (zooplankton growth efficiency) and $K$ (environmental carrying capacity of phytoplankton), which can effectively promote the research process of the plankton bloom. However, a clear understanding of the mechanisms that cause the plankton blooms is still lacking. Hence, by establishing a differential equation model to study the ecological problems and a suitable model to study the dynamic relationship between the phytoplankton and zooplankton is still very important. In the work of [15] and [16], Stomp et al. and Rhee et al. have indicated that there exist many factors affecting the dynamics of plankton.

The functional response is one of the factors affecting the dynamic properties of plankton. Sklaski and Gilliam [17] pointed out that predator-dependent functional responses such as described by Beddington-DeAngelis, Crowley-Martin, and Hassel-Varley can provide a better description of predator feeding over a range of predator-prey abundance settings. In the above three functional responses, the Crowley-Martin [18] functional response is predator dependent and the per capita feeding rate is given by

$$
f(x, y)=\frac{\omega x}{1+k_{1} x+k_{2} y+k_{1} k_{2} x y},
$$

where $\omega, k_{1}$, and $k_{2}$ are positive parameters that describe the effects of the capture rate, handling time, and the magnitude of interference among predators, respectively, on the feeding rate. It is obvious that the following two cases are possible. (1) If $k_{1}>0$ and $k_{2}=0$, then the response reduces to a Michaelis-Menten (or Holling type II) functional response [19]. (2) If $k_{1}=0$ and $k_{2}=0$, then the Crowley-Martin response reduces to a linear massaction (or Holling type I) functional response [20].

It is interesting to study the dynamic properties of plankton systems with different functional responses [21, 22]. To the best of our knowledge, few studies of phytoplanktonzooplankton systems have applied a Crowley-Martin functional response. Based on the above discussion and the previous work of [23], we consider the following system:

$$
\left\{\begin{array}{l}
\frac{d P(t)}{d t}=r P(t)\left(1-\frac{P(t)}{k}\right)-\frac{\omega P(t) Z(t)}{1+k_{1} P(t)+k_{2} Z(t)+k_{1} k_{2} P(t) Z(t)} \\
\frac{d Z(t)}{d t}=\gamma \frac{\omega P(t) Z(t)}{1+k_{1} P(t)+k_{2} Z(t)+k_{1} k_{2} P(t) Z(t)}-\mu Z(t) .
\end{array}\right.
$$

For this system, in order to investigate the dynamics of the phytoplankton and zooplankton, we present the following major assumptions:

$\left(\mathrm{I}_{1}\right)$ It is assumed that $P(t)$ and $Z(t)$ are the concentrations of phytoplankton and zooplankton populations, respectively.

$\left(\mathrm{I}_{2}\right)$ It is assumed that $k\left(u g l^{-1}\right)$ is the carrying capacity, $r\left(d a y^{-1}\right)$ is the maximum growth rate, $\mu\left(d a y^{-1}\right)$ is the zooplankton death rate and $\gamma$ is the conversion efficiency [23].

$\left(\mathrm{I}_{3}\right)$ It is assumed that $\omega$ is the effects of the capture rate, $k_{1}$ is the handing time and $k_{2}$ is the magnitude of interference among predator (zooplankton) [18]. 
$\left(\mathrm{I}_{4}\right)$ From a biological point of view, all the parameters in (2) assume only positive values and will be considered as constants throughout our discussion.

Let $\bar{Z}=Z, \bar{P}=k P$, and $\bar{t}=r t$. Dropping the bars for simplicity, then (2) leads to the following dimensionless equations:

$$
\left\{\begin{array}{l}
\frac{d P(t)}{d t}=P(t)(1-a P(t))-\frac{b P(t) Z(t)}{1+c P(t)+d Z(t)+e P(t) Z(t)}=P(t) f_{1}(P(t), Z(t)), \\
\frac{d Z(t)}{d t}=\frac{g P(t) Z(t)}{1+c P(t)+d Z(t)+e P(t) Z(t)}-h Z(t)=Z(t) f_{2}(P(t), Z(t)) .
\end{array}\right.
$$

With $P(0)=P_{0}>0$ and $Z(0)=Z_{0}>0$, where $a=\frac{1}{k^{2}}, b=\frac{\omega}{r}, c=\frac{k_{1}}{k}, d=k_{2}, e=\frac{k_{1} k_{2}}{k}, h=\frac{\mu}{r}$, $g=\frac{\gamma \omega}{k}$.

On the other hand, it is well known that biological systems governed by delay-differential equations exhibit richer dynamics than ordinary differential systems [24-29]. Many ordinary differential models of plankton ignore the zooplankton from birth to have the ability to hunt and breed, it takes some time. Hence, a time delay factor is taken into account in the ecosystem so it will be more realistic. That is to say, in order to better understand the mechanisms that determine the plankton, it is necessary to study the dynamic behaviors for a phytoplankton-zooplankton system with consider the effect of a time delay. In recent years, the impact of different delays (such as maturation and gestation etc.) on plankton ecosystems has been extensively studied [30-36]. In this work, we assume that reproduction in a zooplankton population after predating on phytoplankton is not instantaneous, but is arbitrated by some constant time lag $\tau$ because of phytoplankton handling and digesting. Under the same basic assumptions as taken for system (3), the dimensionless form of the delayed phytoplankton-zooplankton system is

$$
\left\{\begin{array}{l}
\frac{d P(t)}{d t}=P(t)(1-a P(t))-\frac{b P(t) Z(t)}{1+c P(t)+d Z(t)+e P(t) Z(t)} \\
\frac{d Z(t)}{d t}=\frac{g P(t-\tau) Z(t-\tau)}{1+c P(t-\tau)+d Z(t-\tau)+e P(t-\tau) Z(t-\tau)}-h Z(t)
\end{array}\right.
$$

subject to the initial conditions $P_{0}=\zeta_{1}(\theta)>0, Z_{0}=\zeta_{2}(\theta)>0, \theta \in[-\tau, 0]$, where $\zeta_{i} \in$ $C\left([-\tau, 0] \rightarrow R_{+}\right)$for $i=1,2$ are given functions and $\tau$ is a positive constant.

The main purpose of this paper is to study the dynamic behaviors of system (3) and (4), especially, how the time delay affects the plankton system. We shall also compare all possible dynamics between the non-delayed system (3) and its corresponding delayed system (4). The remainder of the paper is organized as follows. In Section 2, we consider results for the non-delayed system (3), and we discuss the properties of positivity and boundedness, conditions for the existence of a positive equilibrium as well as the condition for the persistence, and the stability of various equilibrium points. Section 3 presents analogous results for the delayed system (4) and considers the stability and direction of the Hopf bifurcation. In Section 4, we present some numerical results supporting our analytical findings. Section 5 discusses the results and draws some conclusions.

\section{Non-delayed system}

In this section, our analysis shows the positivity and boundedness of the solutions, dissipativity, the persistence, local and global stability of both zooplankton-free equilibrium and positive equilibrium points for system (3). 


\subsection{Positivity and boundedness of solutions}

Let $R_{+}$denote the set of all non-negative real numbers and $R_{+}^{n}=\left\{P \in R^{n}: P=\left(P_{1} \cdots P_{n}\right)\right\}$, where $\left\{P_{i} \in R_{+}, \forall i=1,2, \ldots, n\right\}$. If we denote the function on the right-hand side of (3), by $F=\left(F_{1}, F_{2}\right)$, it is clear that $F \in C^{1}\left(R_{+}^{2}\right)$. Thus, $F: R_{+}^{2} \rightarrow R^{2}$ is locally Lipschitz on $R_{+}^{2}=$ $((P, Z): P \geq 0, Z \geq 0)$. Hence, the fundamental theorem of the existence and uniqueness ensures the existence and uniqueness of a solution for (3) with the given initial conditions. The state space for the system is a non-negative cone $R_{+}^{2}=\{(P, Z): P \geq 0, Z \geq 0\}$.

Lemma 2.1 The positive quadrant $\left(\operatorname{Int}\left(R_{+}^{2}\right)\right)$ is invariant for system (3).

Now we consider the conservation of overall energy or biomass flow, and we search for the existence of some region in the dynamical space within which system (3) is bounded.

Lemma 2.2 ([37]) Consider $s(t)$ as an absolutely continuous function satisfying the differential inequality

$$
\frac{d(s(t))}{d t}+u_{1} s(t) \leq u_{2} \quad \text { s.t. } t>0
$$

where $\left(u_{1}, u_{2}\right) \in R^{2}, u_{1} \neq 0$. Then for all $t \geq \hat{T} \geq 0$ we have

$$
s(t) \leq \frac{u_{2}}{u_{1}}-\left(\frac{u_{2}}{u_{1}}-s(\hat{T})\right) e^{-u_{1}(t-\hat{T})}
$$

Theorem 2.3 All the solutions of (3) starting in $R_{+}^{2}$ are confined to the region $D=$ $\left\{(P(t), Z(t)) \in R_{+}^{2}: 0 \leq P(t) \leq \frac{1}{a}, 0 \leq \psi(t) \leq \frac{(h+1)^{2}}{4 a h}\right\}$ as $t \rightarrow \infty$ for all positive initial values $(P(0), Z(0)) \in R_{+}^{2}$, where

$$
\psi(t)=P(t)+\frac{b}{g} Z(t)
$$

Proof We define the function

$$
\psi(t)=P(t)+\frac{b}{g} Z(t)
$$

Differentiating $\psi(t)$ with respect to time $t$ along the solution for system (2), we obtain

$$
\begin{aligned}
\frac{d \psi(t)}{d t}= & \frac{d P(t)}{d t}+\frac{b}{g} \frac{d Z(t)}{d t} \\
= & P(t)(1-a P(t))-\frac{b P(t) Z(t)}{1+c P(t)+d Z(t)+e P(t) Z(t)} \\
& +\frac{b}{g}\left(\frac{g P(t) Z(t)}{1+c P(t)+d Z(t)+e P(t) Z(t)}-h Z(t)\right) \\
= & -h\left(P(t)+\frac{b}{g} Z(t)\right)-\left(a P\left(t^{2}-(h+1) P(t)\right)\right. \\
\leq & -h \psi(t)+\frac{(h+1)^{2}}{4 a} .
\end{aligned}
$$


Thus, we have

$$
\frac{d \psi(t)}{d t}+h \psi(t) \leq \frac{(h+1)^{2}}{4 a}
$$

Using Lemma 2.2, we have $\psi(t) \leq \frac{(h+1)^{2}}{4 a h}$ as $t \rightarrow+\infty$. Therefore, all the solutions for (3) are uniformly bounded with an ultimate bound.

\subsection{Dissipativity and permanence}

In this section, we analyze the dissipativity, persistence, and permanence behavior of system (3). To prove our results, we first present the following definitions.

Definition 2.4 ([38]) System (3) is said to be weakly persistent if every solution $(P(t), Z(t))$ satisfies two conditions:

(1) $P(t) \geq 0, Z(t) \geq 0, \forall t \geq 0$.

(2) $\lim \sup _{t \rightarrow+\infty} P(t)>0, \lim \sup _{t \rightarrow+\infty} Z(t)>0$.

System (3) is said to be strongly persistent if every solution $(P(t), Z(t))$ satisfies the following condition along with the first condition for the weak persistence: $\liminf _{t \rightarrow+\infty} P(t)>$ $0, \liminf _{t \rightarrow+\infty} Z(t)>0$.

Definition 2.5 ([38]) System (3) is said to be permanent if there exists positive constants $m$ and $M$, with $0<m<M$ such that

$$
\begin{aligned}
& \min \left\{\liminf _{t \rightarrow+\infty} P(t), \liminf _{t \rightarrow+\infty} Z(t)\right\} \geq m, \\
& \max \left\{\limsup _{t \rightarrow+\infty} P(t), \limsup _{t \rightarrow+\infty} Z(t)\right\} \leq M,
\end{aligned}
$$

for all solutions $(P(t), Z(t))$ of system (3) with positive initial values. System (3) is said to be non-permanent if there is a positive solution $(P(t), Z(t))$ of system (3) such that

$$
\min \left\{\liminf _{t \rightarrow+\infty} P(t), \liminf _{t \rightarrow+\infty} Z(t)\right\}=0 .
$$

Lemma 2.6 ([39]) If $P>0, q>0$ and $\frac{d u}{d t} \leq(\geq) u(t)(q-p u(t)), u\left(t_{0}\right)>0$, then we have $\lim \sup _{t \rightarrow+\infty} u(t) \leq \frac{q}{p}\left(\liminf _{t \rightarrow+\infty} u(t) \geq \frac{q}{p}\right)$. As the dependent variables are positive, from the phytoplankton equation in system (3), we have

$$
\frac{d P(t)}{d t} \leq P(t)(1-a P(t))
$$

Using Lemma 2.6,

$$
\limsup _{t \rightarrow+\infty} P(t) \leq \frac{1}{a} \equiv m_{1}
$$

Thus, for arbitrary $\varepsilon_{1}>0$ there exists a positive real number $T_{1}$ such that

$$
P(t) \leq m_{1}+\varepsilon_{1}, \quad \forall t \geq T_{1}
$$


It is easy to see that

$$
\frac{d P(t)}{d t} \geq P(t)\left(\left(1-\frac{b}{d}\right)-a P(t)\right)
$$

Applying Lemma 2.6, we have

$$
\liminf _{t \rightarrow+\infty} P(t) \geq \frac{d-b}{a d} \equiv m_{2} \quad \text { provided } d>b
$$

For arbitrary $\varepsilon_{2}>0$ there exists a positive real number $T_{2}$ such that

$$
P(t) \geq m_{2}-\varepsilon_{2}, \quad \forall t \geq T_{2}
$$

From the zooplankton equation in system (3), we have

$$
\begin{aligned}
\frac{d Z(t)}{d t} & \leq Z(t)\left(\frac{g\left(m_{1}+\varepsilon_{1}\right)}{1+c\left(m_{2}-\varepsilon_{2}\right)+d Z(t)+e\left(m_{2}-\varepsilon_{2}\right) Z(t)}-h\right) \\
& =Z(t)\left(\frac{\left(m_{1}+\varepsilon_{1}\right) g-h-c h\left(m_{2}-\varepsilon_{2}\right)-Z(t)\left(d h+e h\left(m_{2}-\varepsilon_{2}\right)\right)}{1+c\left(m_{2}-\varepsilon_{2}\right)+d Z(t)+e\left(m_{2}-\varepsilon_{2}\right) Z(t)}\right) \\
& \leq Z(t)\left(\frac{\left(m_{1}+\varepsilon_{1}\right) g-h-c h\left(m_{2}-\varepsilon_{2}\right)-Z(t)\left(d h+e h\left(m_{2}-\varepsilon_{2}\right)\right)}{1+c\left(m_{2}-\varepsilon_{2}\right)}\right) .
\end{aligned}
$$

Applying Lemma 2.6 yields

$$
\limsup _{t \rightarrow+\infty} Z(t) \leq \frac{m_{1} g-h-c h m_{2}}{d h+e h m_{2}} \equiv Q_{1} \quad \text { provided } h<\frac{m_{1} g}{1+c m_{2}}
$$

Similarly, we can obtain

$$
\liminf _{t \rightarrow+\infty} Z(t) \geq \frac{m_{2} g-h-c h m_{1}}{d h+e h m_{1}} \equiv Q_{2} \quad \text { provided } h<\frac{m_{2} g}{1+c m_{1}}
$$

The above results can be summarized in the following theorem.

Theorem 2.7 For system (3), if $h<\frac{g m_{1}}{1+c m_{2}}$, then

$$
\begin{aligned}
& \limsup _{t \rightarrow+\infty} P(t) \leq \frac{1}{a} \equiv m_{1}, \\
& \limsup _{t \rightarrow+\infty} Z(t) \leq \frac{m_{1} g-h-c h m_{2}}{d h+e h m_{2}} \equiv Q_{1} .
\end{aligned}
$$

Theorem 2.8 For system (3), if $d>b$ and $h<\frac{g m_{2}}{1+c m_{1}}$, then

$$
\begin{aligned}
& \liminf _{t \rightarrow+\infty} P(t) \geq \frac{d-b}{a d} \equiv m_{2}, \\
& \liminf _{t \rightarrow+\infty} Z(t) \geq \frac{g m_{2}-h-c h m_{1}}{d h+e h m_{1}} \equiv Q_{2} .
\end{aligned}
$$


Remark 2.9 Under Definition 2.4, Theorem 2.7 results, whereby system (3) is weakly persistent (dissipative), holding provided $h<\frac{m_{1} g}{1+c m_{2}}$. Theorem 2.8 along with Definition 2.4 ensures that system (3) is strongly persistent provided the conditions $d>b$ and $h<\frac{g m_{2}}{1+c m_{1}}$ hold.

The conditions $d>b, h<\frac{g m_{1}}{1+c m_{2}}$, and $h<\frac{g m_{2}}{1+c m_{1}}$ ensure that $m_{2}>0, Q_{1}>0$, and $Q_{2}>0$, respectively. Since $m_{1}>0$ and $\frac{g m_{1}}{1+c m_{2}}>\frac{g m_{2}}{1+c m_{2}}$, when $h<\frac{g m_{2}}{1+c m_{1}}$ holds, this ensures that $Q_{1}>0$ and $Q_{2}>0$. Thus, we arrive at the following result.

Theorem 2.10 System (3) is permanent if it satisfies any of the following two conditions: $\left(\mathrm{H}_{1}\right) d>b,\left(\mathrm{H}_{2}\right) h<\frac{g m_{2}}{1+c m_{1}}$.

\subsection{Biomass equilibrium}

We consider the following three equilibrium solutions of system (3):

(i) The trivial equilibrium $E^{0}=(0,0)$.

(ii) The boundary equilibrium $E^{1}=\left(\frac{1}{a}, 0\right)$.

(iii) The coexistence equilibrium (interior equilibrium) $E^{*}=\left(P^{*}, Z^{*}\right)$.

Equilibria $E^{0}$ and $E^{1}$ always exist, and the coexistence equilibrium $E^{*}=\left(P^{*}, Z^{*}\right)$ is the point of intersection of zero growth rate isoclines for phytoplankton (i.e. when $\frac{d P(t)}{d t}=0$ ) and zooplankton (i.e. when $\frac{d Z(t)}{d t}=0$ ), given by

$$
\left\{\begin{array}{l}
1-a P-\frac{b Z}{1+c P+d Z+e P Z}=0, \\
\frac{g P}{1+c P+d Z+e P Z}-h=0,
\end{array}\right.
$$

where $Z=\frac{g P(1-a P)}{b h}$, and $P$ is the root of the following equation:

$$
P^{3}+A_{1} P^{2}+A_{2} P+A_{3}=0
$$

where

$$
A_{1}=\frac{a d g-e g}{a g e}, \quad A_{2}=\frac{g b-c h b-d g}{a g e}, \quad A_{3}=-\frac{h b}{a g e}<0 .
$$

From Lemma 2.2 of Song et al. [40], since $A_{3}<0$, we see that equation (6) has at least one positive root. We only consider that equation (6) has one positive root denoted by $P^{*}$. Furthermore, for this value of $P^{*}$, the corresponding value of $Z^{*}$ is given by $Z^{*}=\frac{g P^{*}\left(1-a P^{*}\right)}{b h}$.

\subsection{Dynamical behavior: stability analysis}

In this section, we deal with the local stability, global stability, and bifurcation of system (3).

2.4.1 Behavior of system (3) around $E^{0}=(0,0)$

The variational matrix for system (3) at $E_{0}$ takes the form

$$
J\left(E^{0}\right)=\left(\begin{array}{cc}
1 & 0 \\
0 & -h
\end{array}\right) .
$$

We observe that the Jacobian matrix of system (3) at $E^{0}$ has eigenvalues 1 and $-h$. Therefore, system (3) is always unstable around $E_{0}$, which is in fact a saddle point. 


\subsubsection{Behavior of system (3) around $E^{1}=\left(\frac{1}{a}, 0\right)$}

The variational matrix for system (3) at $E^{1}$ takes the form

$$
J\left(E^{1}\right)=\left(\begin{array}{cc}
-1 & -\frac{b}{a+c} \\
0 & \frac{g}{a+c}-h
\end{array}\right) .
$$

The eigenvalues of the variational matrix $J\left(E^{1}\right)$ at the equilibrium solution $E^{1}$ are -1 and $\frac{g}{a+c}-h$. Hence, for $h>\frac{g}{a+c}$ system (3) is stable around $E_{1}$, for which the $P-Z$ plane is at stable manifold. Conversely, for $h<\frac{g}{a+c}$, system (3) is always unstable around $E^{1}$, which is, in fact, a saddle point.

From the above analysis, we have the following result.

Theorem 2.11 For system (3):

(i) $E^{0}$ is a saddle point.

(ii) $E^{1}$ is a saddle point if $h<\frac{g}{a+c}$, and it is a stable node if $h>\frac{g}{a+c}$.

Theorem 2.12 The equilibrium solution $E^{1}=\left(\frac{1}{a}, 0\right)$ is globally asymptotically stable if $h \geq \frac{g}{a}$.

We prove the result for the delayed system. The proof for system (3) is similar.

\subsubsection{Behavior of system (3) around $E^{*}=\left(P^{*}, Z^{*}\right)$}

Let $P(t)=P^{*}+x(t), Z(t)=Z^{*}+y(t)$. Then the linearized system for (3) around the positive equilibrium $E^{*}=\left(P^{*}, Z^{*}\right)$ is

$$
\left\{\begin{array}{l}
\frac{d x(t)}{d t}=P^{*} f_{1 P} x(t)+P^{*} f_{1 Z} y(t), \\
\frac{d y(t)}{d t}=Z^{*} f_{2 P} x(t)+Z^{*} f_{2 Z} y(t) .
\end{array}\right.
$$

The variational matrix for linearization of system (3) around $E^{*}$ after simplification using the equilibrium equations for system (3) is given by

$$
J\left(E^{*}\right)=\left(\begin{array}{ll}
J_{11} & J_{12} \\
J_{21} & J_{22}
\end{array}\right)
$$

where

$$
\begin{aligned}
& J_{11}=P^{*} f_{1 P}=-a P^{*}+\frac{b Z^{*}\left(c P^{*}+e P^{*} Z^{*}\right)}{\left(1+c P^{*}+d Z^{*}+e P^{*} Z^{*}\right)^{2}}, \\
& J_{12}=P^{*} f_{1 Z}=-\frac{b P^{*}\left(1+c P^{*}\right)}{\left(1+c P^{*}+d Z^{*}+e P^{*} Z^{*}\right)^{2}}<0, \\
& J_{21}=Z^{*} f_{2 P}=\frac{g Z^{*}\left(1+d Z^{*}\right)}{\left(1+c P^{*}+d Z^{*}+e P^{*} Z^{*}\right)^{2}}>0, \\
& J_{22}=Z^{*} f_{2 Z}=-\frac{g P^{*}\left(d Z^{*}+e P^{*} Z^{*}\right)}{\left(1+c P^{*}+d Z^{*}+e P^{*} Z^{*}\right)^{2}}<0 .
\end{aligned}
$$

The characteristic polynomial for $J\left(E^{*}\right)$ is $\lambda^{2}-\operatorname{tr}\left(J\left(E^{*}\right)\right) \lambda+\operatorname{det}\left(J\left(E^{*}\right)\right)$, where $\operatorname{tr}$ denotes trace and det stands for determinant. Applying the Routh-Hurwitz criteria, the characteristic polynomial will have either negative real roots or a pair of complex conjugate root 
with negative real part if $\operatorname{tr}\left(J\left(E^{*}\right)\right)<0$ and $\operatorname{det}\left(J\left(E^{*}\right)\right)>0$. Since $\operatorname{tr}\left(J\left(E^{*}\right)\right)=J_{11}+J_{22}$ and $\operatorname{det}\left(J\left(E^{*}\right)\right)=J_{11} J_{22}-J_{12} J_{21}$, we have $\operatorname{tr}\left(J\left(E^{*}\right)\right)<0$ and $\operatorname{det}\left(J\left(E^{*}\right)\right)>0$ if $J_{11}<0$, in other words, system (3) is stable in a small neighborhood of the non-trivial equilibrium $E^{*}$ if

$$
a>\frac{b Z^{*}\left(c+e Z^{*}\right)}{\left(1+c P^{*}+d Z^{*}+e P^{*} Z^{*}\right)^{2}},
$$

that is, if

$$
f_{1 P}<0
$$

Furthermore, we have the equilibrium equation (see the phytoplankton equation for system (3))

$$
1+c P^{*}+d Z^{*}+e P^{*} Z^{*}=\frac{b Z^{*}}{1-a P^{*}} .
$$

Using (9), equation (8) takes the form

$$
a b Z^{*}>\left(c+e Z^{*}\right)\left(1-a P^{*}\right)^{2} .
$$

Solving (9) for $Z^{*}$, we have

$$
Z^{*}=\frac{\left(1+c P^{*}\right)\left(1-a P^{*}\right)}{b-\left(1-a P^{*}\right)\left(d+e P^{*}\right)} .
$$

Using (11), then inequality (10) takes the form

$$
\frac{a b\left(1+c P^{*}\right)\left(1-a P^{*}\right)}{b-\left(1-a P^{*}\right)\left(d+e P^{*}\right)}>\left(c+e Z^{*}\right)\left(1-a P^{*}\right)^{2}
$$

From (11), we have $b-\left(1-a P^{*}\right)\left(d+e P^{*}\right)>0$ and $P^{*} \leq \frac{1}{a}$. Thus, equation (12) must hold provided

$$
\left(1+c P^{*}\right)\left(a b-e\left(1-a P^{*}\right)\right)>c\left(b-\left(1-a P^{*}\right)\left(d+e P^{*}\right)\right) .
$$

After some simple algebraic manipulations, equation (13) gives

$$
(a b c-c a d+a e) P^{*}+a b-c d+c d-e>0 .
$$

Hence, if $b>d$ and $a b>c b-c d+e$, then (14) is satisfied. Thus, we have the following result.

Theorem 2.13 If $b>d$ and $a b>c b-c d+e$ hold, then the positive equilibrium is locally asymptotically stable.

2.4.4 Global stability of the interior equilibrium $E^{*}=\left(P^{*}, Z^{*}\right)$ of system (3)

Theorem 2.14 If $\left(\mathrm{H}_{1}\right)-\left(\mathrm{H}_{2}\right)$ hold and $\left(1+e Q_{1}\right)\left(1-a P^{*}\right)<a$, then the interior equilibrium solution $E^{*}$ is globally asymptotically stable. 
Proof We prove Theorem 2.14 by constructing a suitable Lyapunov functional. Consider the functional $V(P, Z): R_{+}^{2} \rightarrow R$ such that

$$
V(P, Z)=V_{1}(P)+u V_{2}(Z)
$$

where $V_{1}(P)=\left(P-P^{*}-P^{*} \ln \left(\frac{P}{P^{*}}\right)\right), V_{2}(Z)=\left(Z-Z^{*}-Z^{*} \ln \left(\frac{Z}{Z^{*}}\right)\right)$, and $u$ is a positive constant to be defined below. We evaluate the time derivative of the positive definite scalar valued function (15) along the solutions for system (3):

$$
\begin{aligned}
\frac{d V_{1}}{d t} & =\left(P-P^{*}\right) \frac{d P}{P d t} \\
& =\left(P-P^{*}\right)\left(-a\left(P-P^{*}\right)+\frac{b Z^{*}}{1+c P^{*}+d Z^{*}+e P^{*} Z^{*}}-\frac{b Z}{1+c P+d Z+e P Z}\right), \\
\frac{d V_{2}}{d t} & =u\left(Z-Z^{*}\right) \frac{d Z}{Z d t} \\
& =u\left(Z-Z^{*}\right)\left(\frac{g P}{1+c P+d Z+e P Z}-\frac{g P^{*}}{1+c P^{*}+d Z^{*}+e P^{*} Z^{*}}\right) .
\end{aligned}
$$

We define $N(P, Z)=(1+c P+d Z+e P Z)\left(1+c P^{*}+d Z^{*}+e P^{*} Z^{*}\right)$. Differentiating (15) and inserting values of $\frac{d V_{1}}{d t}$ and $\frac{d V_{2}}{d t}$, algebraic manipulation yields

$$
\begin{aligned}
\frac{d V}{d t}= & \left(P-P^{*}\right)\left\{-a\left(P-P^{*}\right)+\frac{b\left(Z^{*}-Z\right)+b c\left(P Z^{*}-Z P^{*}\right)+b e Z Z^{*}\left(P-P^{*}\right)}{N(P, Z)}\right\} \\
& +u\left(Z-Z^{*}\right)\left\{\frac{g\left(P-P^{*}\right)+g d\left(P Z^{*}-Z P^{*}\right)+g e P P^{*}\left(Z^{*}-Z\right)}{N(P, Z)}\right\} \\
= & \left(P-P^{*}\right) \\
& \times\left\{-a\left(P-P^{*}\right)+\frac{b\left(Z^{*}-Z\right)+b c\left(Z^{*}\left(P-P^{*}\right)-P^{*}\left(Z-Z^{*}\right)\right)+b e Z Z^{*}\left(P-P^{*}\right)}{N(P, Z)}\right\} \\
& +u\left(Z-Z^{*}\right)\left\{\frac{g\left(P-P^{*}\right)+g d\left(Z^{*}\left(P-P^{*}\right)-P^{*}\left(Z-Z^{*}\right)\right)+g e P P^{*}\left(Z^{*}-Z\right)}{N(P, Z)}\right\} \\
= & \left(-a+\frac{b c Z^{*}+b e Z Z^{*}}{N(P, Z)}\right)\left(P-P^{*}\right)^{2}+\frac{\left(g d u Z^{*}+g u-\left(b+P^{*}\right)\right)}{N(P, Z)}\left(P-P^{*}\right)\left(Z-Z^{*}\right) \\
& -\frac{\left(g d P^{*} u+u g e P P^{*}\right)}{N(P, Z)}\left(Z-Z^{*}\right)^{2} .
\end{aligned}
$$

Using Theorems 2.7 and 2.8 and choosing $u$ such that $u\left(g d Z^{*}+g\right)=b+P^{*}$, we have

$$
\frac{d V}{d t}<-\left[a-\frac{b Z^{*}\left(c+e Q_{1}\right)}{N(P, Z)}\right]\left(P-P^{*}\right)^{2}-\left[\frac{u\left(g d P^{*}+g e P P^{*}\right)}{N(P, Z)}\right]\left(Z-Z^{*}\right)^{2} .
$$

The coefficient for $\left(Z-Z^{*}\right)^{2}$ in (16) is always negative under the hypotheses of Theorem 2.14. After inserting value of $N(P, Z)$, the coefficient for $\left(P-P^{*}\right)^{2}$ takes the following form:

$$
\begin{aligned}
& -a+\frac{b Z^{*}\left(c+e Q_{1}\right)}{(1+c P+d Z+e P Z)\left(1+c P^{*}+d Z^{*}+e P^{*} Z^{*}\right)} \\
& \leq-a+\frac{b Z^{*}\left(c+e Q_{1}\right)}{1+c P^{*}+d Z^{*}+e P^{*} Z^{*}}=-a+\left(c+e Q_{1}\right)\left(1-a P^{*}\right) .
\end{aligned}
$$


We conclude that if the hypotheses of Theorem 2.14 are satisfied, then $\frac{d V}{d t}<0$ along all the trajectories in $R_{+}^{2}$ except $E^{*}=\left(P^{*}, Z^{*}\right)$. Thus, the functional $V(P, Z)$ satisfies all the properties of a Lyapunov functional. Therefore, $E^{*}=\left(P^{*}, Z^{*}\right)$ is globally asymptotically stable under the conditions of Theorem 2.14. This completes the proof.

\section{Delayed system}

In this section, we analytically prove positivity and boundedness of the solutions, the persistence, local and global stability, existence of the Hopf bifurcation by analyzing the associated characteristic transcendental equation for the model with a delay. Furthermore, we also discuss stability and direction of the Hopf bifurcation by means of the normal form method and center manifold theory.

\subsection{Positivity and boundedness of system (4)}

First we prove the positivity of system (4). We can write the phytoplankton equation in system (4) as

$$
\frac{d P}{P}=\left(1-a P-\frac{b Z}{1+c P+d Z+e P Z}\right) d t
$$

Integrating from 0 to $t$, we obtain the following result:

$$
P(t)=P(0) e^{\left[\int_{0}^{t}\left\{1-a P(\varphi)-\frac{b Z(\varphi)}{1+c P(\varphi)+d Z(\varphi)+e P(\varphi) Z(\varphi)}\right\} d \varphi\right]},
$$

which implies that $P(t)>0$ for all $t$ when $P(0)>0$.

Using the zooplankton equation in system (4), we can write

$$
Z(t)=Z(0) e^{\left[\int_{0}^{t}\left\{\frac{g P(\varphi-\tau) Z(\varphi-\tau)}{Z(\varphi)[1+c P(\varphi-\tau)+d Z(\varphi-\tau)+e P(\varphi-\tau) Z(\varphi-\tau)]}-h\right\} d \varphi\right]},
$$

which implies that $Z(t)>0$ for all $t$ when $Z(0)>0$. Hence the interior of the first quadrant is an invariant set for the delayed system (4).

Theorem 3.1 All solutions for system (4) starting in $R_{+}^{2}$ are confined to the region $D^{*}=$ $\left\{(P, Z) \in R_{+}^{2}: 0 \leq \phi(t) \leq \frac{(h+1)^{2}}{4 a h}\right\}$ as $t \rightarrow+\infty$ for all positive initial values $\left(P_{0}(\theta), Z_{0}(\theta)\right) \in R_{+}^{2}$, where $\phi(t)=P(t-\tau)+\frac{b}{g} Z(t)$.

Proof We define the function $\phi(t)=P(t-\tau)+\frac{b}{g} Z(t)$. Differentiating $\phi(t)$ with respect to time $t$ along the solution for system (4), we obtain

$$
\begin{aligned}
\frac{d \phi(t)}{d t}= & \frac{d P(t-\tau)}{d t}+\frac{b}{g} \frac{d Z(t)}{d t} \\
= & P(t-\tau)(1-a P(t-\tau))-\frac{b P(t-\tau) Z(t-\tau)}{1+c P(t-\tau)+d Z(t-\tau)+e P(t-\tau) Z(t-\tau)} \\
& +\frac{b}{g}\left\{\frac{g P(t-\tau) Z(t-\tau)}{1+c P(t-\tau)+d Z(t-\tau)+e P(t-\tau) Z(t-\tau)}-h Z(t)\right\} \\
= & P(t-\tau)-a P^{2}(t-\tau)-\frac{b h}{g} Z(t)
\end{aligned}
$$




$$
\begin{aligned}
& =-h\left(P(t-\tau)+\frac{b}{g} Z(t)\right)+(h+1) P(t-\tau)-a P^{2}(t-\tau) \\
& \leq-h \phi(t)+\frac{(h+1)^{2}}{4 a} .
\end{aligned}
$$

Thus, we have

$$
\frac{d \phi(t)}{d t}+h \phi(t) \leq \frac{(h+1)^{2}}{4 a} .
$$

Using Lemma 2.2, we have $\phi(t) \leq \frac{(h+1)^{2}}{4 a h}$. Thus all the solutions for system (4) are uniformly bounded with an ultimate bound.

\subsection{Permanence}

Now we establish the persistence and permanence behavior of the delayed model using the positivity of the dependent variables.

In Section 2, we showed the following relations for the phytoplankton equation in system (4):

$$
\limsup _{t \rightarrow+\infty} P(t) \leq \frac{1}{a} \equiv m_{1}
$$

and

$$
\liminf _{t \rightarrow+\infty} P(t) \geq \frac{d-b}{a d} \equiv m_{2} .
$$

From the zooplankton equation in system (4), we have

$$
\begin{aligned}
\frac{d Z}{d t} & \leq \frac{g m_{1} Z(t-\tau)}{1+c m_{2}+d Z(t-\tau)+e m_{2} Z(t-\tau)}-h Z(t) \\
& \leq \frac{g m_{1}}{d+e m_{2}}-h Z(t) .
\end{aligned}
$$

Solving (17), we have

$$
\limsup _{t \rightarrow+\infty} Z(t) \leq \frac{g m_{1}}{\left(d+e m_{2}\right) h} .
$$

Because $\frac{g m_{1}}{\left(d+e m_{2}\right) h}>\frac{g m_{1}-h-c m_{2} h}{\left(d+e m_{2}\right) h} \equiv Q_{1}$, we obtain lim $\sup _{t \rightarrow+\infty} Z(t) \leq Q_{1}$.

Similarly, we have

$$
\begin{aligned}
\frac{d Z}{d t} & \geq \frac{g m_{2} Z(t-\tau)}{1+c m_{1}+d Z(t-\tau)+e m_{1} Z(t-\tau)}-h Z(t) \\
& \geq \frac{g m_{2}}{d+e m_{1}}-h Z(t) .
\end{aligned}
$$

Solving (18), we have

$$
\liminf _{t \rightarrow+\infty} Z(t) \geq \frac{g m_{2}}{\left(d+e m_{1}\right) h}>\frac{g m_{2}-h-c m_{1} h}{\left(d+e m_{1}\right) h} \equiv Q_{2} .
$$

The above results can be summarized as follows. 
Theorem 3.2 Any positive solution $\{P(t), Z(t)\}$ of system (4) satisfies

$$
\begin{aligned}
& m_{2} \leq \liminf _{t \rightarrow+\infty} P(t) \leq \limsup _{t \rightarrow+\infty} P(t) \leq m_{1}, \\
& Q_{2} \leq \liminf _{t \rightarrow+\infty} Z(t) \leq \limsup _{t \rightarrow+\infty} Z(t) \leq Q_{1},
\end{aligned}
$$

when $d>b$ and $h<\frac{m_{2} g}{1+c m_{1}}$.

Using the definitions of limsup and liminf, we can claim that there exist four positive constants $t_{i}(i=1,2,3,4)$ such that

$$
\begin{aligned}
& m_{2} \leq P(t), \quad \forall t \geq t_{1}, \quad P(t) \leq m_{1}, \quad \forall t>t_{2}, \\
& Q_{2} \leq Z(t), \quad \forall t \geq t_{3}, \quad Z(t) \leq Q_{1}, \quad \forall t>t_{4} .
\end{aligned}
$$

If we define $N^{*}=\min \left\{m_{2}, Q_{2}\right\}, L^{*}=\max \left\{m_{1}, Q_{1}\right\}$, and $T^{*}=\max \left\{t_{1}, t_{2}, t_{3}, t_{4}\right\}$, then we have $N^{*}<\{P(t), Z(t)\}<L^{*}$ for $t>T^{*}$, and we can state the permanence result for system (3).

Theorem 3.3 The delayed system (4) is permanent if $d>b$ and $h<\frac{g m_{2}}{1+c m_{1}}$.

\subsection{Local stability and Hopf-bifurcation analysis}

In this section we discuss the local stability of the positive equilibrium $E^{*}$ and the boundary equilibrium $E^{1}$ for system (4) and establish the existence of the Hopf bifurcation at $E^{*}$. We recall the following result, which provides the conditions for the absence of a delayinduced switch from stability to instability.

Theorem 3.4 ([41]) The set of necessary and sufficient conditions for $E^{*}$ to be asymptotically stable for all $\tau \geq 0$ is as follows:

(i) The real parts of all the roots of $D(\lambda, 0)=0$ are negative.

(ii) For any real $Z$ and any $\tau \geq 0, D(i Z, 0) \neq 0$, where $i=\sqrt{-1}$, and $D(\lambda, \tau)$ denotes the characteristic equation associated with (4).

We can rewrite system (4) as $\frac{d W(t)}{d t}=F(W(t), W(t-\tau))$, where $W(t)=[P(t), Z(t)]^{T}$ and $W(t-\tau)=[P(t-\tau), Z(t-\tau)]^{T}$. Let $P(t)=P^{*}+x(t), Z(t)=Z^{*}+y(t)$. Then, linearizing system (4) about the interior equilibrium $E^{*}=\left(P^{*}, Z^{*}\right)$, we have

$$
\frac{d \varphi(t)}{d t}=G U(t)+H U(t-\tau)
$$

where $G=\left(\frac{\partial F}{\partial W(t)}\right)_{W^{*}}, H=\left(\frac{\partial F}{\partial W(t-\tau)}\right)_{W^{*}}, W^{*}=\left(P^{*}, Z^{*}\right)$, and $\varphi(t)=(x(t), y(t))$.

Thus, the variational matrix for system (4) at $E^{*}$ is given by $J\left(E^{*}\right)=G+H e^{-\lambda \tau}$, where

$$
\begin{aligned}
& G=\left(\begin{array}{cc}
j_{11} & j_{12} \\
0 & j_{23}
\end{array}\right), \\
& H=\left(\begin{array}{cc}
0 & 0 \\
j_{21} & j_{22}
\end{array}\right),
\end{aligned}
$$


with

$$
\begin{aligned}
& j_{11}=-a P^{*}+\frac{b Z^{*}\left(c P^{*}+e P^{*} Z^{*}\right)}{\left(1+c P^{*}+d Z^{*}+e P^{*} Z^{*}\right)^{2}}, \\
& j_{12}=-\frac{b P^{*}\left(1+c P^{*}\right)}{\left(1+c P^{*}+d Z^{*}+e P^{*} Z^{*}\right)^{2}}, \\
& j_{21}=\frac{g Z^{*}\left(1+d Z^{*}\right)}{\left(1+c P^{*}+d Z^{*}+e P^{*} Z^{*}\right)^{2}}, \\
& j_{22}=\frac{g P^{*}\left(1+c P^{*}\right)}{\left(1+c P^{*}+d Z^{*}+e P^{*} Z^{*}\right)^{2}}, \\
& j_{23}=-h .
\end{aligned}
$$

The variational matrix of system (4) at $E^{1}$ is given by

$$
J\left(E^{1}\right)=\left(\begin{array}{cc}
-1 & -\frac{b}{a+c} \\
0 & -h+\frac{g}{a+c} e^{-\lambda \tau}
\end{array}\right)
$$

and its corresponding characteristic equation is

$$
\operatorname{det}\left(\begin{array}{cc}
-1-\lambda & -\frac{b}{a+c} \\
0 & \frac{g}{a+c} e^{-\lambda \tau}-h-\lambda
\end{array}\right)=0
$$

where $\lambda=-1$ is a negative eigenvalue. Hence, we only need to concentrate on the equation

$$
\lambda=-h+\frac{g}{a+c} e^{-\lambda \tau}
$$

It is clear that when $\tau=0$ and $h>\frac{g}{a+c}$, the equilibrium point $E^{1}$ is locally asymptotically stable. Substituting $\lambda=i \omega$ into equation (20) and equating the real and imaginary parts, we obtain

$$
\cos \omega \tau=\frac{h(a+c)}{g}, \quad \sin \omega \tau=-\frac{\omega(a+c)}{g} .
$$

Solving the above two equations, we have

$$
\omega^{2}=\left(\frac{g}{a+c}\right)^{2}-h^{2}
$$

Equation (22) has a positive root $\omega_{+}$if $\frac{g}{a+c}>h$. Thus, there is a positive constant $\hat{\tau}$ such that, for $\tau>\hat{\tau}, E^{1}$ becomes unstable.

The characteristic equation for system (4) at the positive equilibrium $E^{*}$ takes the form

$$
D(\lambda, \tau)=\operatorname{det}\left(G+H e^{-\lambda \tau}-\lambda I\right)=\operatorname{det}\left(\begin{array}{cc}
j_{11}-\lambda & j_{12} \\
j_{21} e^{-\lambda \tau} & -\frac{g}{b} j_{12} e^{-\lambda \tau}-h-\lambda
\end{array}\right)=0 .
$$

Then we have the following transcendental equation:

$$
\lambda^{2}+\left(h-j_{11}\right) \lambda-h j_{11}=j_{12}\left(j_{21}+\frac{g}{b} j_{11}-\frac{g}{b} \lambda\right) e^{-\lambda \tau},
$$


which has been extensively studied [42, 43]. Here $D(\lambda, 0)=\lambda^{2}+\left(h-j_{11}\right) \lambda-h j_{11}-j_{12}\left(j_{21}+\right.$ $\left.\frac{g}{b} j_{11}-\frac{g}{b} \lambda\right)$. Hence, the constant term in $D(\lambda, 0)=0$ is negative if $h+j_{12} \frac{g}{b}>j_{11}$ and $j_{12}\left(j_{21}+\right.$ $\left.\frac{g}{b} j_{11}\right)+h j_{11}<0$. Therefore, regardless of the sign for $\lambda, D(\lambda, 0)=0$ always has a unique positive root. Consequently, Theorem 3.4 fails to ensure the stability of system (4) with positive delay $\tau>0$. We now determine the conditions under which the system undergoes a Hopf bifurcation near the coexistence equilibrium $E^{*}$ and the local stability of $E^{*}$. We take the discrete time delay $\tau$ as the bifurcation parameter. We know that the sign for the real parts of the solution for (23) characterize the stability behavior of $E^{*}$. Therefore, putting $\lambda=\mu+i \omega$ into (23) and separating the real and imaginary parts, we obtain the following system of equations:

$$
\begin{aligned}
\mu^{2} & -\omega^{2}+h \mu-j_{11} \mu-h j_{11} \\
& =\left(j_{12} j_{21}+\frac{g}{b} j_{12} j_{11}-\frac{g}{b} j_{12} \mu\right) e^{-\mu \tau} \cos \omega \tau-\frac{g}{b} j_{12} e^{-\mu \tau} \omega \sin \omega \tau, \\
2 \mu \omega & +\omega h-j_{11} \omega \\
& =-\frac{g}{b} j_{12} \omega e^{-\mu \tau} \cos \omega \tau-e^{-\mu \tau}\left(j_{12} j_{21}+\frac{g}{b} j_{12} j_{11}-\frac{g}{b} j_{12} \mu\right) \sin \omega \tau .
\end{aligned}
$$

The necessary condition for a change in stability of the interior equilibrium point $E^{*}$ is that equation (23) should have purely imaginary roots, that is, stability switches for increasing $\tau$ in $I=\left[0, \tau^{*}\right)$ may occur only with a pair of roots $\lambda= \pm i \omega(\tau)$. Let $\tau=\hat{\tau}$ be the particular magnitude of $\tau$ for which $\mu(\hat{\tau})=0$ and $\omega(\hat{\tau})=\hat{\omega}$. Hence, to obtain the stability criterion, substituting $\tau=\hat{\tau}, \mu=0$, and $\omega=\hat{\omega}$ in (19), we obtain

$$
\begin{aligned}
& \hat{\omega}^{2}+h j_{11}=-\left(j_{12} j_{21}+\frac{g}{b} j_{12} j_{11}\right) \cos \hat{\omega} \hat{\tau}+\frac{g}{b} j_{12} \hat{\omega} \sin \hat{\omega} \hat{\tau}, \\
& \left(j_{11}-h\right) \hat{\omega}=\frac{g}{b} j_{12} \hat{\omega} \cos \hat{\omega} \hat{\tau}+\sin \hat{\omega} \hat{\tau}\left(j_{12} j_{21}+\frac{g}{b} j_{12} j_{11}\right) .
\end{aligned}
$$

Solving (25) for $\cos \hat{\omega} \hat{\tau}$ and $\sin \hat{\omega} \hat{\tau}$, we obtain

$$
\begin{aligned}
& \cos \hat{\omega} \hat{\tau}=\frac{\hat{\omega}^{2}\left(j_{11}-h\right) \frac{g}{b}-\left(\hat{\omega}^{2}+h j_{11}\right)\left(j_{21}+\frac{g}{b} j_{11}\right)}{j_{12}\left[\left(j_{21}+\frac{g}{b} j_{11}\right)^{2}+\left(\frac{g}{b} \hat{\omega}\right)^{2}\right]} \\
& \sin \hat{\omega} \hat{\tau}=\frac{\hat{\omega}\left(j_{11}-h\right)\left(j_{21}+\frac{g}{b} j_{11}\right)+\left(\hat{\omega}^{2}+h j_{11}\right) \frac{g}{b} \hat{\omega}}{j_{12}\left[\left(j_{21}+\frac{g}{b} j_{11}\right)^{2}+\left(\frac{g}{b} \hat{\omega}\right)^{2}\right]} .
\end{aligned}
$$

By squaring and adding the above two equations, we obtain a third order algebraic equation in $\hat{\omega}$ of the form

$$
a_{0} \xi^{3}+3 a_{1} \xi^{2}+3 a_{2} \xi+a_{3}=0
$$

where

$$
\begin{aligned}
& \xi=\hat{\omega}^{2}, \quad a_{0}=\frac{g^{2}}{b^{2}}, \\
& 3 a_{1}=2 \frac{g}{b}\left(j_{11} j_{21}+\frac{g}{b} j_{11}^{2}-h j_{21}\right)+\left(\frac{g}{b} h+j_{21}\right)^{2}-\frac{g^{4}}{b^{4}} j_{12}^{2},
\end{aligned}
$$




$$
\begin{aligned}
& 3 a_{2}=\left(j_{21} j_{11}+\frac{g}{b} j_{11}^{2}-h j_{21}\right)^{2}+2\left(\frac{g}{b} h+j_{21}\right)\left(h j_{11} j_{21}+\frac{g}{b} j_{11}^{2} h\right)-2\left(j_{12} \frac{g}{b}\left(j_{21}+\frac{g}{b} j_{11}\right)\right)^{2}, \\
& a_{3}=\left(h j_{11} j_{21}+\frac{g}{b} j_{11}^{2} h\right)^{2}-j_{12}^{2}\left(j_{21}+\frac{g}{b} j_{11}\right)^{4} .
\end{aligned}
$$

Let $\xi$ be a real positive root of the cubic equation

$$
a_{0} x^{3}+3 a_{1} x^{2}+3 a_{2} x+a_{3}=0
$$

Letting $v=a_{0} x+a_{1}$, then equation (28) can be rewritten as

$$
h(v)=v^{3}+3 e_{1} v+e_{2},
$$

where

$$
e_{1}=a_{0} a_{2}-a_{1}^{2}, \quad e_{2}=a_{0}^{2} a_{3}-3 a_{0} a_{1} a_{2}+2 a_{1}^{3}
$$

From Lemma 2.2 of Wang et al. [44] we know that: $\left(\mathrm{s}_{1}\right)$ If $e_{2}<0$, equation (29) has one positive root. ( $\left.\mathrm{s}_{2}\right)$ If $e_{2}>0$ and $e_{1}<0$, equation (29) has two positive roots when $e_{2}^{2}+4 e_{1}^{3}<0$ and has a positive root of multiplicity two when $e_{2}^{2}+4 e_{1}^{2}=0$. ( $\left.\mathrm{s}_{3}\right)$ If $e_{2}=0$ and $e_{1}<0$, equation (29) has one positive root.

The required value of $\hat{\omega}$, for which equation (23) has a pair of purely imaginary roots is the positive root of (27). From (26), the critical magnitude of the delay parameter corresponding to $\hat{\omega}$ is given by

$$
\hat{\tau}_{n}=\frac{1}{\hat{\omega}} \arccos \frac{\hat{\omega}^{2} \frac{g}{b}\left(j_{11}-h\right)-\left(\hat{\omega}^{2}+h j_{11}\right)\left(j_{21}+\frac{g}{b} j_{11}\right)}{j_{12}\left[\left(j_{21}+\frac{g}{b} j_{11}\right)^{2}+\left(\frac{g}{b} \hat{\omega}\right)^{2}\right]}+\frac{2 n \pi}{\hat{\omega}}
$$

for $n=0,1,2, \ldots$ If $E^{*}$ is locally asymptotically stable for $\tau=0$, then by Butler's lemma (see Appendix 2 in [45]), $E^{*}$ remains stable for $\tau<\hat{\tau}$. The transversality condition for the Hopf bifurcation at $\tau=\hat{\tau}$ is $\left[\frac{d \mu}{d \tau}\right]_{\tau=\hat{\tau}}>0$. Differentiating (24) with respect to $\tau$ and setting $\tau=\hat{\tau}, \omega=\hat{\omega}$, and $\mu=0$, we obtain

$$
\beta_{1}\left[\frac{d \mu}{d \tau}\right]_{\tau=\hat{\tau}}-\alpha_{1}\left[\frac{d \omega}{d \tau}\right]_{\tau=\hat{\tau}}=\beta_{2}, \quad \beta_{1}\left[\frac{d \mu}{d \tau}\right]_{\tau=\hat{\tau}}+\alpha_{1}\left[\frac{d \omega}{d \tau}\right]_{\tau=\hat{\tau}}=\alpha_{2}
$$

where

$$
\begin{aligned}
& \beta_{1}=h-j_{11}+j_{12} \cos \hat{\omega} \hat{\tau}\left(\frac{g}{b}+j_{21} \hat{\tau}+\frac{g}{b} j_{11} \hat{\tau}\right)-\frac{g}{b} \hat{\omega} \hat{\tau} j_{12} \sin \hat{\omega} \hat{\tau}, \\
& \alpha_{1}=-\left(j_{12} \sin \hat{\omega} \hat{\tau}\left(\frac{g}{b}+j_{21} \hat{\tau}+\frac{g}{b} j_{11} \hat{\tau}\right)+\frac{g}{b} \hat{\omega} \hat{\tau} j_{12} \cos \hat{\omega} \hat{\tau}-2 \hat{\omega}\right), \\
& \beta_{2}=-j_{12}\left(\frac{g}{b} \hat{\omega}^{2} \cos \hat{\omega} \hat{\tau}+\hat{\omega} \sin \hat{\omega} \hat{\tau}\left(j_{21}+\frac{g}{b} j_{11}\right)\right), \\
& \alpha_{2}=j_{12}\left(\frac{g}{b} \hat{\omega}^{2} \sin \hat{\omega} \hat{\tau}-\hat{\omega} \cos \hat{\omega} \hat{\tau}\left(j_{21}+\frac{g}{b} j_{11}\right)\right) .
\end{aligned}
$$


Thus, we have

$$
\left[\frac{d \mu}{d \tau}\right]_{\tau=\hat{\tau}}=\frac{\beta_{2} \beta_{1}+\alpha_{2} \alpha_{1}}{\beta_{1}^{2}+\alpha_{1}^{2}} .
$$

From (30) it is obvious that the transversality condition $\left[\frac{d \mu}{d \tau}\right]_{\tau=\hat{\tau}}>0$ for the occurrence of the Hopf bifurcation at $\tau=\hat{\tau}$ is well satisfied provided $\beta_{2} \beta_{1}+\alpha_{2} \alpha_{1}>0$. Summarizing, we have established the following result for switching the stability behavior of system (4) around $E^{*}$.

\section{Theorem 3.5}

(i) Let $E^{*}$ be locally asymptotically stable for system (4) with $\tau=0$ and let $-h j_{11}-j_{12}\left(j_{21}+\frac{g}{b} j_{11}\right)<0$. Then there exists $\tau=\hat{\tau}$ such that $E^{*}$ is locally asymptotically stable for $\tau<\hat{\tau}$ and unstable for $\tau>\hat{\tau}$, where $\hat{\tau}>0$ is the smallest value for which there is a solution to equation (23) for which the real part is zero. Furthermore, as $\tau$ exceeds $\hat{\tau}$, $E^{*}$ bifurcates into periodic solutions, provided $\beta_{2} \beta_{1}+\alpha_{2} \alpha_{1}>0$.

(ii) If $h+j_{12} \frac{g}{b}>j_{11}, j_{12}\left(j_{21}+\frac{g}{b} j_{11}\right)+h j_{11}<0$, and $\left(\mathrm{s}_{1}\right)$ or $\left(\mathrm{s}_{2}\right)$ or $\left(\mathrm{s}_{3}\right)$ hold, then $E^{*}$ for system (4) is locally asymptotically stable for all $\tau>0$.

\subsection{Global stability of system (4) around $E^{1}$ and $E^{*}$}

We discuss the global stability of $E^{1}$ and $E^{*}$. Let $(P(t), Z(t))$ be any positive solution for (4) and let $E^{1}=\left(\frac{1}{a}, 0\right)=(\bar{P}, 0)$. We consider the function

$$
V_{1}=P-\bar{P}-\bar{P} \ln \frac{P}{\bar{P}}+\frac{b}{g} Z
$$

The time derivative of (31) along the positive solution of system (4) is

$$
\begin{aligned}
\frac{d V_{1}}{d t}= & \frac{P-\bar{P}}{P} \frac{d P}{d t}+\frac{b}{g} \frac{d Z}{d t} \\
= & (P-\bar{P})\left[(1-a P)-\frac{b Z}{1+c P+d Z+e P Z}\right] \\
& +\frac{b P(t-\tau) Z(t-\tau)}{1+c P(t-\tau)+d Z(t-\tau)+e P(t-\tau) Z(t-\tau)}-\frac{b h}{g} Z \\
= & -a(P-\bar{P})^{2}-\frac{b P Z}{1+c P+d Z+e P Z}+\frac{\frac{b}{a} Z}{1+c P+d Z+e P Z} \\
& +\frac{b P(t-\tau) Z(t-\tau)}{1+c P(t-\tau)+d Z(t-\tau)+e P(t-\tau) Z(t-\tau)}-\frac{b h}{g} Z .
\end{aligned}
$$

We introduce a functional $V_{2}$ for (32) such that

$$
V_{2}=V_{1}+b \int_{t-\tau}^{t} \frac{P(s) Z(s)}{1+c P(s)+d Z(s)+e P(s) Z(s)} d s .
$$

Calculating $\frac{d V_{2}}{d t}$ along the solution for system (4), we have

$$
\begin{aligned}
\frac{d V_{2}}{d t}= & \frac{d V_{1}}{d t}+\frac{b P Z}{1+c P+d Z+e P Z} \\
& -\frac{b P(t-\tau) Z(t-\tau)}{1+c P(t-\tau)+d Z(t-\tau)+d Z(t-\tau)+e P(t-\tau) Z(t-\tau)}
\end{aligned}
$$




$$
\begin{aligned}
& =-a(P-\bar{P})^{2}+\frac{\frac{b}{a} Z}{1+c P+d Z+e P Z}-\frac{b h}{g} Z \\
& \leq-a(P-\bar{P})^{2}+\left(\frac{b}{a}-\frac{b}{g} h\right) Z .
\end{aligned}
$$

If $h>\frac{g}{a}$ or $h=\frac{g}{a}$, then from (35) we have $\frac{d V_{2}}{d t}<0$ except at $(P(t), Z(t))=(\bar{P}, 0)$ and also $\frac{d V_{2}}{d t}=0$ if and only if $(P(t), Z(t))=(\bar{P}, 0)$ (see the equation of $\left.(34)\right)$. Hence LyapunovLasalle's invariance principle implies the global asymptotic stability of $E^{1}$.

We summarize the result in the following theorem.

Theorem 3.6 The equilibrium solution $E^{1}=\left(\frac{1}{a}, 0\right)$ for system (4) is globally asymptotically stable if $h \geq \frac{g}{a}$.

Theorem 3.7 If $\left(\mathrm{H}_{1}\right)-\left(\mathrm{H}_{2}\right), a>b Z^{*}\left(c+e Q_{1}\right)$, and the conditions

(i) $W=\frac{g Q_{2} P^{*}\left(d+e m_{2}\right)}{Q_{1} \varphi_{1}}-\frac{Q_{1}\left(1+g d Z^{*}\right)}{2 Q_{2} \varphi_{2}}-\frac{g m_{1}\left(1+c P^{*}\right)}{2 Q_{2} \varphi_{2}}>0$,

(ii) $Q_{2} \varphi_{2}\left(b\left(1+c P^{*}\right)\right)^{2} K^{2}-4 Q_{2} W \varphi_{1}^{2}\left(a-b c Z^{*}-b e Q_{1} Z^{*}\right) K$

$$
-2 W \varphi_{1} \varphi_{2} g m_{1}\left(1+c P^{*}\right) \leq 0,
$$

hold, then system (4) has a globally asymptotically stable equilibrium $E^{*}$.

Proof Consider the set $\eta=\left\{(P, Z): m_{2}<P<m_{1}, Q_{2}<Z<Q_{1}\right\}$. It is clear that $\eta$ is a compact and bounded region in $R_{+}^{2}$ and is at a positive distance from the coordinate axes. There exists $T$, such that, for $t>T$, every solution for system (4) with $\tau>0$ enters and remains in region $\eta$.

Using the equations for system (3), we can express system (4) as

$$
\begin{aligned}
\frac{d P}{d t}= & P\left(-a\left(P-P^{*}\right)+\frac{b Z^{*}}{1+c P^{*}+d Z^{*}+e P^{*} Z^{*}}-\frac{b Z}{1+c P+d Z+e P Z}\right), \\
\frac{d Z}{d t}= & Z\left(\frac{g P(t-\tau) Z(t-\tau)}{Z(1+c P(t-\tau)+d Z(t-\tau)+e P(t-\tau) Z(t-\tau))}\right. \\
& \left.-\frac{g P^{*}}{1+c P^{*}+d Z^{*}+e P^{*} Z^{*}}\right) .
\end{aligned}
$$

Motivated by (36), to study the global stability of the interior solution $E^{*}\left(P^{*}, Z^{*}\right)$, we introduce the functional $V(t)$ such that

$$
\begin{aligned}
V(t)= & u_{1} \int_{0}^{P-P^{*}} \frac{s}{s+P^{*}} d s+u_{2} \int_{0}^{Z-Z^{*}} \frac{s}{s+Z^{*}} d s \\
& +R \int_{t-\tau}^{t}\left(P(s)-P^{*}\right)^{2} d s,
\end{aligned}
$$

where $u_{1}$ and $u_{2}$ are positive constants defined below and $R=\frac{u_{2} g m_{1}\left(1+c P^{*}\right)}{2 Q_{2} \varphi_{1}}$, with $\varphi_{1}=$ $\varphi\left(m_{1}, Q_{1}\right), \varphi_{2}=\varphi\left(m_{2}, Q_{2}\right)$, and $\varphi(P, Z)=\left(1+c P^{*}+d Z^{*}+e P^{*} Z^{*}\right)(1+c P+d Z+e P Z)$.

Differentiating $V(t)$ along the solution for system (36) and using

$$
P Z^{*}-P^{*} Z=Z^{*}\left(P-P^{*}\right)+P^{*}\left(Z^{*}-Z\right),
$$




$$
\begin{aligned}
& Z^{*} P(t-\tau)-P^{*} Z=Z^{*}\left(P(t-\tau)-P^{*}\right)+P^{*}\left(Z^{*}-Z\right), \\
& a b \leq \frac{a^{2}+b^{2}}{2},
\end{aligned}
$$

we have

$$
\begin{aligned}
& \frac{d V}{d t}=u_{1}\left(P-P^{*}\right)\left\{\frac{-a\left(P-P^{*}\right)+b\left(Z^{*}-Z\right)+b c\left(P Z^{*}-Z P^{*}\right)+b e Z Z^{*}\left(P-P^{*}\right)}{\varphi(P, Z)}\right\} \\
& +u_{2}\left(Z-Z^{*}\right)\left\{\frac{g\left(P(t-\tau) Z(t-\tau)-P^{*} Z\right)+g c P^{*} P(t-\tau)(Z(t-\tau)-Z)}{Z \varphi(P(t-\tau), Z(t-\tau))}\right\} \\
& +u_{2}\left(Z-Z^{*}\right)\left\{\frac{g d Z(t-\tau)\left(Z^{*} P(t-\tau)-P^{*} Z\right)+g e P^{*} P(t-\tau) Z(t-\tau)\left(Z^{*}-Z\right)}{Z \varphi(P(t-\tau), Z(t-\tau))}\right\} \\
& +R\left(P(t)-P^{*}\right)^{2}-R\left(P(t-\tau)-P^{*}\right)^{2} \\
& =\frac{\left(u_{1} b c Z^{*}-a u_{1}+u_{1} b e Z Z^{*}\right)\left(P-P^{*}\right)^{2}-\left(b u_{1}+u_{1} b c P^{*}\right)\left(Z-Z^{*}\right)\left(P-P^{*}\right)}{\varphi(P, Z)} \\
& +\left\{\frac{\left(g u_{2} P(t-\tau)+u_{2} g c P^{*} P(t-\tau)\right)(Z(t-\tau)-Z)\left(Z-Z^{*}\right)}{Z \varphi(P(t-\tau), Z(t-\tau))}\right\} \\
& +\left\{\frac{\left(u_{2} Z+u_{2} g d Z^{*} Z(t-\tau)\right)\left(P(t-\tau)-P^{*}\right)\left(Z-Z^{*}\right)}{Z \varphi(P(t-\varphi), Z(t-\tau))}\right\} \\
& -\left\{\frac{\left(u_{2} P^{*} g d Z(t-\tau)+u_{2} g e P^{*} P(t-\tau) Z(t-\tau)\right)\left(Z-Z^{*}\right)^{2}}{Z \varphi(P(t-\tau), Z(t-\tau))}\right\} \\
& +R\left(P(t)-P^{*}\right)^{2}-R\left(P(t-\tau)-P^{*}\right)^{2} \\
& \leq-\left\{\frac{u_{1}\left(a-b c Z^{*}-b e Q_{1} Z^{*}\right)}{\varphi_{2}}-R\right\}\left(P-P^{*}\right)^{2} \\
& -\left\{\frac{b u_{1}\left(1+c P^{*}\right)}{\varphi_{1}}\right\}\left(Z-Z^{*}\right)\left(P-P^{*}\right) \\
& -\left\{\frac{u_{2} g Q_{2} P^{*}\left(d+e m_{2}\right)}{Q_{1} \varphi_{1}}-\frac{u_{2} Q_{1}\left(1+g d Z^{*}\right)}{2 Q_{2} \varphi_{2}}-\frac{g u_{2} m_{1}\left(1+c P^{*}\right)}{2 Q_{2} \varphi_{1}}\right\}\left(Z-Z^{*}\right)^{2} .
\end{aligned}
$$

The right-hand side of the above expression should be considered as a quadratic form in the variables $\left(P-P^{*}\right)$ and $\left(Z-Z^{*}\right)$, that is, negative definite if the matrix $G$ is

$$
G=\left(\begin{array}{cc}
\frac{u_{1}\left(a-b c Z^{*}-b e Q_{1} Z^{*}\right)}{\varphi_{2}}-R & \frac{b u_{1}\left(1+c P^{*}\right)}{2 \varphi_{1}} \\
\frac{b u_{1}\left(1+c P^{*}\right)}{2 \varphi_{1}} & \frac{g Q_{2} P^{*}\left(d+e m_{2}\right)}{Q_{1} \varphi_{1}}-\frac{Q_{1}\left(1+g d Z^{*}\right)}{2 Q_{2} \varphi_{2}}-\frac{g m_{1}\left(1+c P^{*}\right)}{2 Q_{2} \varphi_{2}}
\end{array}\right) .
$$

We define $W=\frac{g Q_{2} P^{*}\left(d+e m_{2}\right)}{Q_{1} \varphi_{1}}-\frac{Q_{1}\left(1+g d Z^{*}\right)}{2 Q_{2} \varphi_{2}}-\frac{g m_{1}\left(1+c P^{*}\right)}{2 Q_{2} \varphi_{2}}$ and $K=\frac{\varphi_{2} g m_{1}\left(1+c P^{*}\right)}{Q_{2} \varphi_{1}\left(a-b c Z^{*}-b e Q_{1} Z^{*}\right)}$. If we choose $u_{1}=K u_{2}$, then the matrix $G$ is positive definite if $a>b Z^{*}\left(c+e Q_{1}\right), W>0$, and $4 W\left\{\frac{K\left(a-b c Z^{*}-b e Q_{1} Z^{*}\right)}{\varphi_{2}}-\frac{g m_{1}\left(1+c P^{*}\right)}{2 Q_{2} \varphi_{1}}\right\} \geq \frac{b\left(1+c P^{*}\right)^{2}}{\varphi_{1}^{2}}$, that is, $Q_{2} \varphi_{2}\left(b\left(1+c P^{*}\right)\right)^{2} K^{2}-4 Q_{2} W \varphi_{1}^{2}(a-$ $\left.b c Z^{*}-b e Q_{1} Z^{*}\right) K-2 W \varphi_{1} \varphi_{2} g m_{1}\left(1+c P^{*}\right) \leq 0$. The result follows.

Remark 3.8 If $h \geq g P^{*}$, the sufficient conditions for global asymptotic stability of the positive solution $E^{*}$ for non-delayed system (3) imply that the interior equilibrium solution $E^{*}$ for the delayed system is globally asymptotic stable if $E^{*}$ for non-delayed system (3) is globally asymptotic stable and conditions (i) and (ii) of Theorem 3.7 hold. 


\subsection{Stability and direction of Hopf bifurcation}

Here we describe the process for computing the direction of the Hopf bifurcation and the stability of bifurcating periodic solutions using the normal form method and center manifold theory introduced by Hassard et al. [46]. Without loss of generality, we always assume that system (4) undergoes a Hopf bifurcation in the state $E^{*}$ for one of the critical values $\tau=\hat{\tau}_{j}=\hat{\tau}$, where $j=1,2,3, \ldots$ and $\pm i \omega$ are the only purely imaginary roots of the characteristic equation at $E^{*}$.

Let $\sigma=\tau-\hat{\tau}$. Then $\sigma=0$ is a Hopf-bifurcation value of system (4). Letting $P=x+P^{*}$, $Z=y+Z^{*}$, and $t=t \tau$, system (4) is transformed into

$$
\begin{aligned}
\dot{x}(t)= & a_{11} x(t)+a_{12} y(t)+\sum_{i+j \geq 2} \frac{1}{i ! j !} F_{i j}^{(1)} x^{i}(t) y^{j}(t), \\
\dot{y}(t)= & a_{21} x(t-\tau)+a_{22} y(t-\tau)+a_{23} y(t) \\
& +\sum_{i+j+l \geq 2} \frac{1}{i ! j ! l !} F_{i j l}^{(2)} x^{i}(t-\tau) y^{j}(t-\tau) y^{l}(t),
\end{aligned}
$$

where

$$
\begin{aligned}
& F^{(1)}=P(1-a P)-\frac{b P Z}{1+c P+d Z+e P Z}, \\
& F^{(2)}=\frac{g P(t-\tau) Z(t-\tau)}{1+c P(t-\tau)+d Z(t-\tau)+e P(t-\tau) Z(t-\tau)}-h Z, \\
& F_{i j}^{(1)}=\left.\frac{\partial^{i+j} F^{(1)}}{\partial P^{i} \partial Z^{j}}\right|_{E^{*}} \\
& F_{i j l}^{(2)}=\left.\frac{\partial^{i+j+l} F^{(2)}}{\partial P^{i}(t-\tau) \partial Z^{j}(t-\tau) \partial Z^{l}}\right|_{E^{*}}, \\
& a_{11}=-P^{*} a+\frac{b Z^{*}\left(c P^{*}+e P^{*} Z^{*}\right)}{\left(1+c P^{*}+d Z^{*}+e P^{*} Z^{*}\right)^{2}}, \\
& a_{12}=-\frac{b P^{*}\left(1+c P^{*}\right)}{\left(1+c P^{*}+d Z^{*}+e P^{*} Z^{*}\right)^{2}}, \\
& a_{21}=\frac{g Z^{*}\left(1+d Z^{*}\right)}{\left(1+c P^{*}+d Z^{*}+e P^{*} Z^{*}\right)^{2}}, \\
& a_{22}=\frac{g P^{*}\left(1+c P^{*}\right)}{\left(1+c P^{*}+d Z^{*}+e P^{*} Z^{*}\right)^{2}}, \\
& a_{23}=-h .
\end{aligned}
$$

We can write equation (37) as the following functional differential equation in $C=$ $C\left([-1,0), R^{2}\right)$ :

$$
\dot{x}(t)=L_{\sigma}\left(x_{t}\right)+F\left(a, x_{t}\right)
$$

where $x(t)=\left(x_{1}(t), x_{2}(t)\right)^{T} \in R^{2}, L_{\sigma}: C \rightarrow R$, and $F: R \times C \rightarrow R$ are given by

$$
L_{\sigma}(\phi)=(\hat{\tau}+\sigma) S_{1} \phi(0)+(\hat{\tau}+\sigma) S_{2} \phi(-1)
$$


and

$$
F(\sigma, \phi)=(\hat{\tau}+\sigma)\left(\begin{array}{c}
\sum_{i+j \geq 2} \frac{1}{i, j ! j} F_{i j}^{(1)} \phi_{1}^{i}(0) \phi_{1}^{j}(0) \\
\sum_{i+j+l \geq 2} \frac{1}{i \cdot j \cdot ! l !} F_{i j l}^{(2)} \phi_{1}^{i}(-1) \phi_{2}^{j}(-1) \phi_{2}^{l}(0)
\end{array}\right),
$$

for which $\phi(\theta)=\left(\phi_{1}(\theta), \phi_{2}(\theta)\right)^{T} \in C, B_{1}, B_{2}$, and $F\left(\sigma, x_{t}\right)$ are defined in the form

$$
\begin{aligned}
& S_{1}=\left(\begin{array}{cc}
j_{11} & j_{12} \\
0 & j_{23}
\end{array}\right), \\
& S_{2}=\left(\begin{array}{cc}
0 & 0 \\
j_{21} & j_{22}
\end{array}\right), \\
& F(\sigma, \phi)=(\hat{\tau}+\sigma)\left(\begin{array}{c}
F_{20}^{(1)} \phi_{1}^{2}(0)+F_{11}^{(1)} \phi_{1}(0) \phi_{2}(0)+F_{02}^{(1)} \phi_{2}^{2}(0) \\
F_{200}^{(2)} \phi_{1}^{2}(-1)+F_{110}^{(2)} \phi_{1}(-1) \phi_{2}(-1)+F_{020}^{(2)} \phi_{2}^{2}(-1)
\end{array}\right) .
\end{aligned}
$$

By the Riesz representation theorem [47], there exists a function $\eta(\theta, \sigma)$ of bounded variation for $\theta \in[-1,0]$ such that

$$
L_{\sigma}(\phi)=\int_{-1}^{0} d \eta(\theta, 0) \phi(\theta), \quad \phi \in C
$$

In fact, we can choose

$$
\eta(\theta, \sigma)=(\hat{\tau}+\sigma) S_{1}=\left(\begin{array}{cc}
j_{11} & j_{12} \\
0 & j_{23}
\end{array}\right) \delta(\theta)-(\hat{\tau}+\sigma)\left(\begin{array}{cc}
0 & 0 \\
j_{21} & j_{22}
\end{array}\right) \delta(\theta+1),
$$

where $\delta$ is the Dirac delta function. For $\phi \in\left([-1,0], R^{2}\right)$, we define

$$
A(\sigma) \phi= \begin{cases}\frac{d \phi(\theta)}{d \theta}, & \text { for } \theta \in[-1,0) \\ \int_{-1}^{0} d \eta(\sigma, v) \phi(v), & \text { for } \theta=0\end{cases}
$$

and

$$
R(\sigma) \phi= \begin{cases}0, & \text { for } \theta \in[-1,0) \\ f(\sigma, \phi), & \text { for } \theta=0\end{cases}
$$

Then system (38) is equivalent to

$$
\dot{x}_{t}=A(\sigma) x_{t}+R(\sigma),
$$

where $x_{t}(\theta)=x(t+\theta)$ for $\theta \in(-1,0]$.

For $\kappa \in C^{1}\left([0,1],\left(R^{2}\right)^{*}\right)$, we define

$$
A^{*} \kappa(v)= \begin{cases}-\frac{d \kappa(v)}{d v}, & \text { for } v \in(0,1] \\ \int_{-1}^{0} d \eta^{T}(t, 0) \phi(-t), & \text { for } v=0\end{cases}
$$


To construct the co-ordinates to describe $C_{0}$ near 0 , for $\kappa \in[-\tau, 0]$, we define the bilinear inner product

$$
\langle\kappa(v), \phi(\theta)\rangle=\bar{\kappa}(0) \phi(0)-\int_{-1}^{0} \int_{\xi=0}^{\theta} \bar{\kappa}(\xi-\theta) d \eta(\theta) \phi(\xi) d \xi,
$$

where $\eta(\theta)=\eta(\theta, 0)$. Then $\langle\kappa, A \phi\rangle=\left\langle A^{*} \kappa, \phi\right\rangle$ and hence $A(0)$ and $A^{*}$ are adjoint operators. From the discussion in the previous subsection and the foregoing assumption, we know that $\pm \hat{\omega} \hat{\tau}$ are eigenvalues of $A(0)$. Hence, they are also eigenvalues of $A^{*}$. We first need to compute the eigenvectors of $A(0)$ and $A^{*}$ corresponding to $i \hat{\omega} \hat{\tau}$ and $-i \hat{\omega} \hat{\tau}$.

We can see that

$$
\begin{aligned}
& q(\theta)=(1, \rho)^{T} e^{i \hat{\omega} \theta} \quad(\theta \in[-1,0)), \\
& q^{*}(s)=D\left(1, \rho^{*}\right) e^{i \hat{\omega} s} \quad(s \in(0, \tau]),
\end{aligned}
$$

are the eigenvectors of $A(0)$ and $A^{*}$ corresponding to $i \hat{\omega} \hat{\tau}$ and $-i \hat{\omega} \hat{\tau}$, where

$$
\begin{aligned}
& \rho=\frac{i \hat{\omega}-a_{11}}{a_{12}}, \quad \rho^{*}=\frac{i \hat{\omega}-a_{11}}{a_{21} e^{-i \hat{\omega} \hat{\imath}}}, \\
& D=\frac{1}{1+\bar{\rho} \rho^{*}-\rho^{*} \hat{\tau}\left(a_{21}+a_{22} \bar{\rho}\right) e^{i \hat{\omega} \hat{\tau}}},
\end{aligned}
$$

such that $\left\langle q^{*}(s), q(\theta)\right\rangle=1$ and $\left\langle q^{*}(s), \bar{q}(\theta)\right\rangle=0$.

Following the same algorithms and using a similar computation process to that of Tripathi et al. [38], we obtain the coefficients for the important parameters:

$$
\begin{aligned}
g_{20}= & \hat{\tau} \bar{D}\left(F_{20}^{(1)}+2 F_{11}^{(1)} \rho+\bar{\sigma}\left(F_{200}^{(2)} e^{-2 i \hat{\omega} \hat{\tau}}+2 F_{110}^{(2)} \rho e^{-2 i \hat{\omega} \hat{\imath}}\right)\right), \\
g_{11}= & \hat{\tau} \bar{D}\left(F_{20}^{(1)}+2 F_{11}^{(1)} \operatorname{Re}\{\rho\}+\bar{\rho}^{*}\left(F_{200}^{(2)}+2 F_{110}^{(2)} \operatorname{Re}\{\rho\}\right)\right), \\
g_{02}= & \hat{\tau} \bar{D}\left(F_{20}^{(1)}+2 F_{11}^{(1)} \bar{\rho}+\bar{\rho}^{*}\left(F_{200}^{(2)} e^{2 i \hat{\omega} \hat{\tau}}+2 F_{110}^{(2)} \bar{\rho} e^{2 i \hat{\omega} \hat{\tau}}\right)\right), \\
g_{21}= & \hat{\tau} \bar{D}\left(F_{11}^{(1)} W_{20}^{(2)}(0)+F_{11}^{(1)} W_{20}^{(1)}(0) \bar{\rho}\right) \\
& +\hat{\tau} \bar{D} \bar{\rho}^{*}\left(F _ { 1 1 0 } ^ { ( 2 ) } \left(W_{20}^{(1)}(-1) \bar{\rho} e^{i \hat{\omega} \hat{\tau}}+W_{20}^{(2)}(-1) e^{i \hat{\omega} \hat{\tau}}\right.\right. \\
& \left.\left.+2 W_{11}^{(1)}(-1) \rho e^{-i \hat{\omega} \hat{\tau}}+2 W_{11}^{(2)}(-1) e^{-i \hat{\omega} \hat{\tau}}\right)\right),
\end{aligned}
$$

where

$$
\begin{aligned}
& W_{20}(\theta)=\frac{i g_{20}}{\hat{\omega} \hat{\tau}} q(0) e^{i \hat{\omega} \hat{\tau} \theta}+\frac{i \bar{g}_{02}}{3 \hat{\omega} \hat{\tau}} \bar{q}(0) e^{-i \hat{\omega} \hat{\tau} \theta}+E_{1} e^{2 i \hat{\omega} \hat{\tau} \theta}, \\
& W_{11}(\theta)=-\frac{i g_{20}}{\hat{\omega} \hat{\tau}} q(0) e^{i \hat{\omega} \hat{\tau}}+\frac{i \bar{g}_{11}}{\hat{\omega} \hat{\tau}} \bar{q}(0) e^{-i \hat{\omega} \hat{\tau}}+E_{2}, \\
& E_{1}=\left(\begin{array}{c}
E_{1}^{(1)} \\
E_{1}^{(2)}
\end{array}\right), \\
& E_{2}=\left(\begin{array}{c}
E_{2}^{(1)} \\
E_{2}^{(2)}
\end{array}\right), \\
& E_{1}^{(1)}=\frac{2}{\Delta}\left|\begin{array}{cc}
c_{1} & -a_{12} \\
c_{2} & 2 i \hat{\omega}+h+2 e Z^{*}+a_{22} e^{-2 i \hat{\omega} \hat{\tau}}
\end{array}\right|
\end{aligned}
$$




$$
\begin{aligned}
& E_{1}^{(2)}=\frac{2}{\Delta}\left|\begin{array}{cc}
2 i \hat{\omega}-a_{11} & c_{1} \\
-a_{21} e^{-2 i \hat{\omega} \hat{\imath}} & c_{2}
\end{array}\right|, \\
& E_{2}^{(1)}=-\frac{2}{\Delta^{*}}\left|\begin{array}{cc}
c_{1} & a_{12} \\
c_{2} & -h-a_{22}
\end{array}\right|, \\
& E_{2}^{(2)}=-\frac{2}{\Delta^{*}}\left|\begin{array}{ll}
a_{11} & d_{1} \\
a_{21} & d_{2}
\end{array}\right|, \\
& \Delta=\left|\begin{array}{cc}
2 i \hat{\omega}-a_{11} & -a_{12} \\
-a_{21} e^{2 i \hat{\omega} \hat{\tau}} & 2 i \hat{\omega}+a_{22} e^{-2 i \hat{\omega} \hat{\imath}}
\end{array}\right|, \\
& \Delta^{*}=-\left.\Delta\right|_{\theta=0, \hat{\omega}=0}, \\
& c_{1}=F_{20}^{(1)}+2 F_{11}^{(1)} \rho+F_{02}^{(1)} \rho^{2}, \\
& c_{2}=F_{200}^{(2)} e^{-2 i \hat{\omega} \hat{\imath}}+2 F_{110}^{(2)} \rho e^{-2 i \hat{\omega} \hat{\imath}}+F_{020}^{(2)} \rho^{2} e^{-2 i \hat{\omega} \hat{\tau}} \text {, } \\
& d_{1}=F_{20}^{(1)}+2 F_{11}^{(1)} \operatorname{Re}(\rho)+F_{02}^{(1)}|\rho|^{2}, \\
& d_{2}=F_{200}^{(2)}+2 F_{110}^{(2)} \operatorname{Re}(\rho)+F_{020}^{(2)}|\rho|^{2} .
\end{aligned}
$$

Consequently, $g_{21}$ can be expressed explicitly. Thus, we can compute the following values:

$$
\begin{aligned}
& c_{1}(0)=\frac{i}{2 \hat{\omega} \hat{\tau}}\left(g_{20} g_{11}-2\left|g_{11}\right|^{2}-\frac{\left|g_{02}\right|^{2}}{3}\right)+\frac{g_{21}}{2}, \\
& \mu_{2}=-\frac{\operatorname{Re}\left\{c_{1}(0)\right\}}{\operatorname{Re} \frac{d \lambda(\hat{\tau})}{d \tau}}, \\
& \beta_{2}=2 \operatorname{Re}\left\{c_{1}(0)\right\} \\
& T_{2}=-\frac{\operatorname{Im}\left\{c_{1}(0)\right\}+\mu_{2} \operatorname{Im}\left\{\frac{d \lambda(\hat{\tau})}{d \tau}\right\}}{\hat{\omega} \hat{\tau}} .
\end{aligned}
$$

According to Hassard et al. [46] and using the quantity analysis above, the Hopfbifurcation properties are determined using the following theorem.

Theorem 3.9 The Hopf-bifurcation properties at the critical value $\tau=\hat{\tau}$ are derived, as follows:

(i) If $\mu_{2}>0(<0)$, the Hopf bifurcation is supercritical (subcritical).

(ii) If $\beta_{2}<0(>0)$, the bifurcated periodic solutions are stable (unstable).

(iii) If $T_{2}>0(<0)$, period of the bifurcating periodic solution increases (decreases).

\section{Numerical simulations}

In the previous sections, a detailed theoretical analysis has been carried out and some interesting results of the research of system have been obtained. In order to prove the feasibility and effectiveness of the theoretical analysis results as well as to provide a more in-depth understanding of the results of the analysis, in particular, the time delay influencing the complex dynamics of phytoplankton-zooplankton system, we perform some numerical simulations. 
The numerical solutions are given considering the following parameter values: $a=1, b=$ $5, c=2, d=6, e=0.001, h=0.3$, and $g=5$. For this choice, it is easy to show that the parameters can satisfy the conditions of Theorem 2.14 when $\tau=0$. And thus the positive equilibrium point $E^{*}=(0.3937050691,0.7956712927)$ has global attractivity, which implies that the phytoplankton and zooplankton populations may have lasting coexistence, as shown in Figure 1. In addition, these parameters can also satisfy the conditions of Theorem 3.7 when we take $\tau=2.5$, and the positive equilibrium point $E^{*}=(0.3937050691,0.7956712927)$ and this also shows global attractivity in the phytoplankton-zooplankton system with a time delay (see Figure 2). Furthermore, it is interesting to compare Figure 1 and Figure 2, especially in Figure 1(c) and Figure 2(c), where it can be seen that the phase diagram of Figure 2 becomes finer, which indicates that the delay does not change the stability of the positive equilibrium point, but it can also accelerate the process of its stability. To further illustrate the point, we plot phase portraits with the same initial value, and when $\tau=2.5$ and $\tau=0$ in Figure 3, and the time series diagram for six different values of delay: 0, 0.5, 2.5, 5, 7.5, and 12 in Figure 4 are taken, this explicitly depicts that the time delay does not affect the stability of the positive equilibrium point.

Next, the direction of the Hopf bifurcation for the corresponding delayed system (4) is also investigated by a numerical simulation. We take $a=1, b=8, c=3.09, d=1.2$, $e=0.0005, h=1.1$, and $g=8$, then it is not difficult to obtain $\hat{\omega}=0.5393409834$, $\beta_{1} \beta_{2}+\alpha_{2} \alpha_{1}>0, \hat{\tau}=0.4422694125$. It is easy to verify that the positive equilibrium
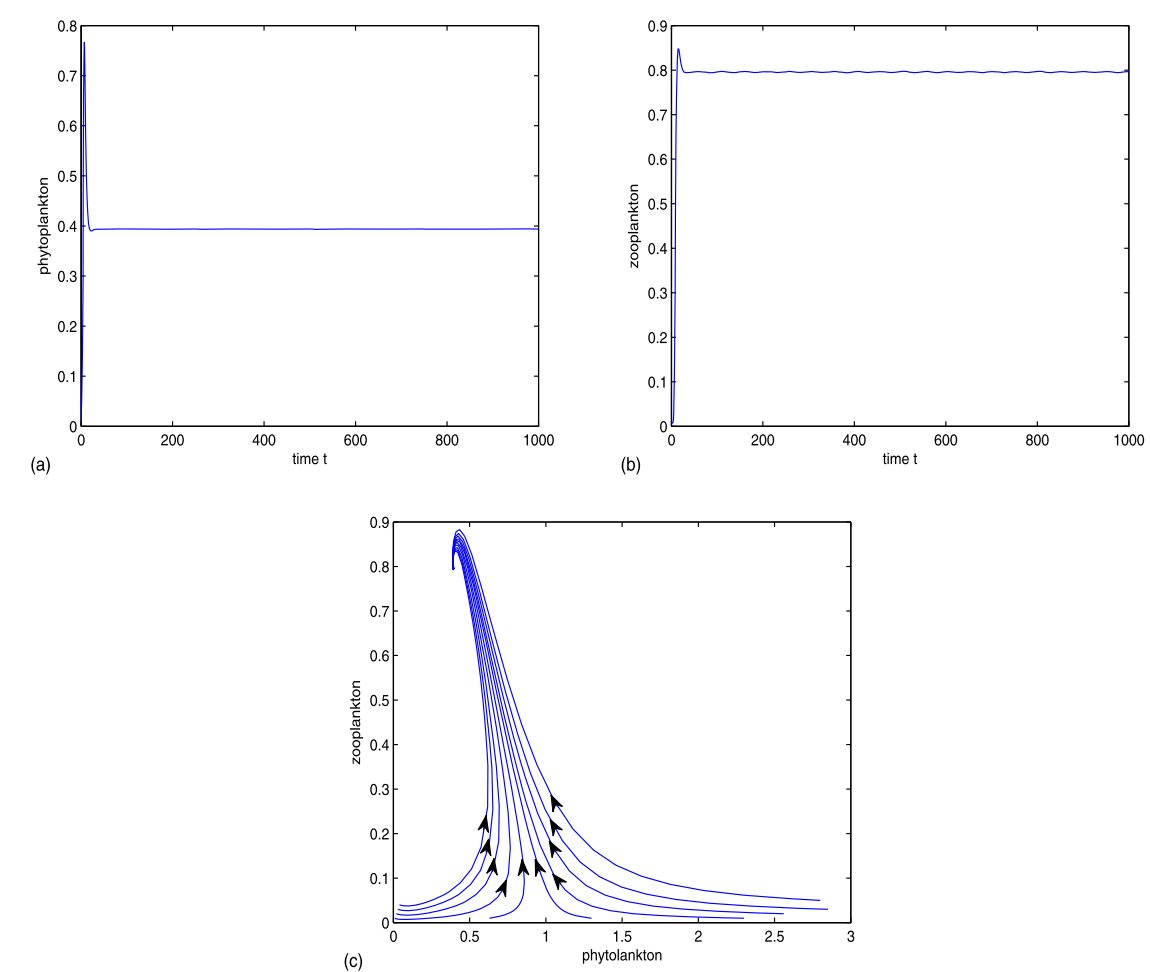

Figure 1 The global attractivity of system (3). (a)-(b) Time series. (c) Phase portrait. We take the parameters $a=1, b=5, c=2, d=6, e=0.001, h=0.3$, and $g=5$, and we obtain ten sets of different initial values $(0.01,0.01),(0.02,0.02),(0.03,0.03),(0.04,0.04),(0.63,0.01),(1.3,0.01),(2.3,0.01),(2.56,0.02),(2.80,0.05)$, and $(2.85,0.03)$. Numerical simulations show for system (3) the positive equilibrium point $E^{*}$ has global attractivity when $\tau=0$. 

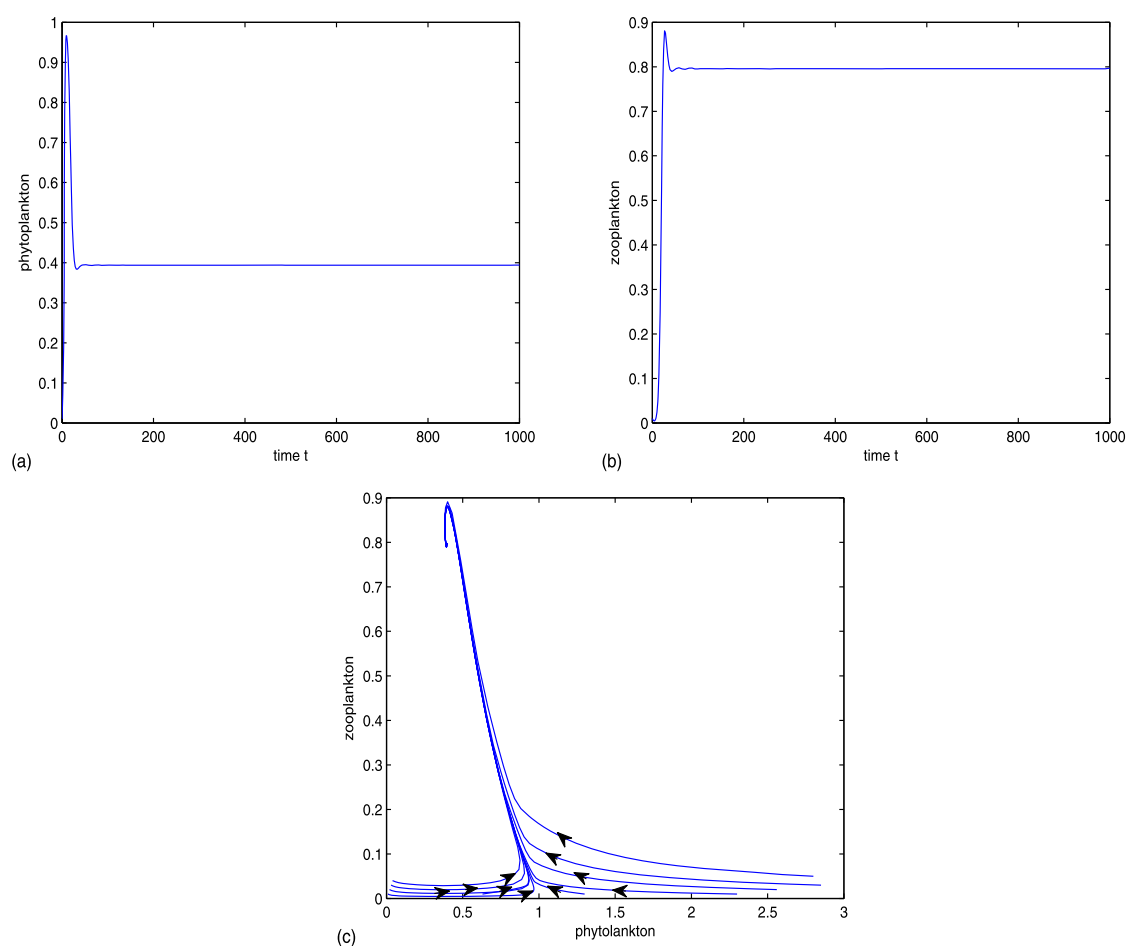

Figure 2 The global attractivity of system (4). (a)-(b) Time series. (c) Phase portrait. The parameters and initial values are the same as given in Figure 1. Numerical simulations show that for system (4) the positive equilibrium point $E^{*}$ has global attractivity when $\tau=2.5$.

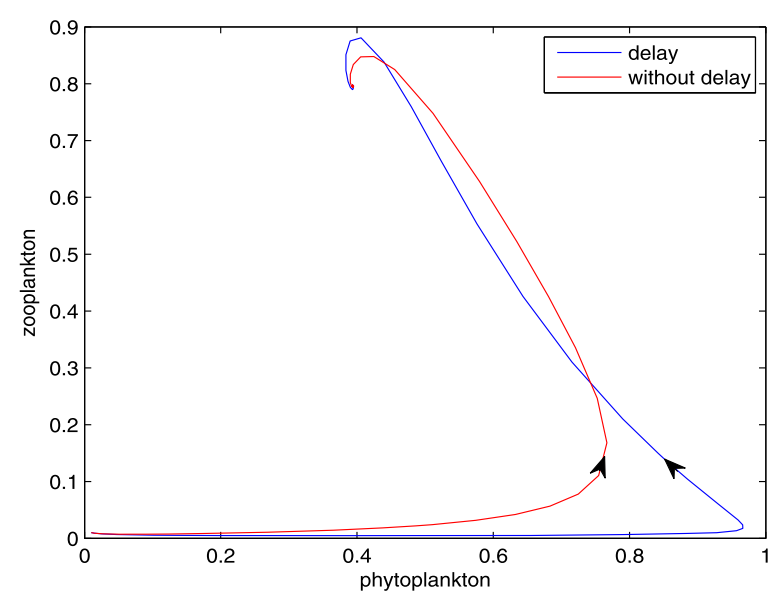

Figure 3 The phase portrait shows the effect of the delay on the stability of the coexistence equilibrium point $E^{*}$.

$E^{*}=(0.2931263460,0.1883666284)$ for system $(4)$ is locally asymptotically stable when $\tau<\hat{\tau}$, the case of $\tau=0.110$ is illustrated in Figure 5. More interesting, when $\tau$ passes through the critical value $\hat{\tau}$, the positive equilibrium $E^{*}=(0.2931263460,0.1883666284)$ for system (4) loses its stability and a Hopf bifurcation occurs. Moreover, there exists a positive period- 1 solution caused by the Hopf bifurcation, the case of $\tau=0.59$ is shown 


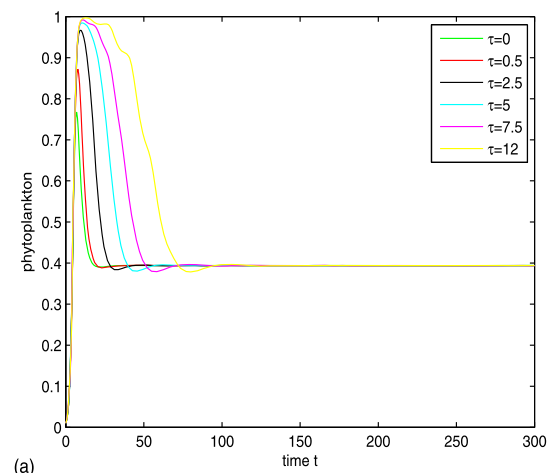

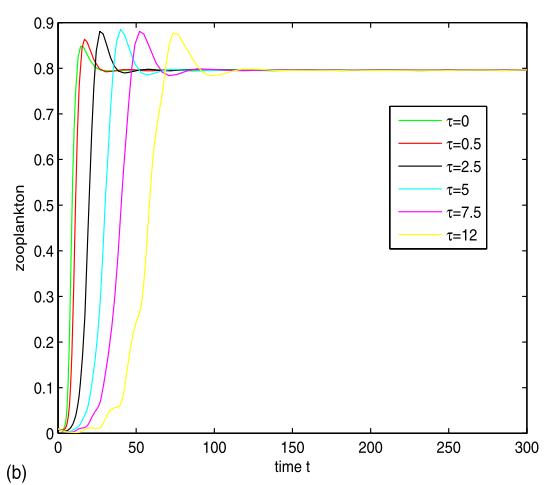

Figure 4 The effects of different time delays on the stability of the solution. We take the same parameters as in Figure 1 and obtain six different values of the delay, 0, 0.5, 2.5, 5, 7.5, and 12. The green line is for $\tau=0$, the red line for $\tau=0.5$, the black line for $\tau=2.5$, the cyan line for $\tau=5$, the pinkish red line for $\tau=7.5$, and the yellow line for $\tau=12$. (a) The time series of the phytoplankton. (b) The time series of the zooplankton.
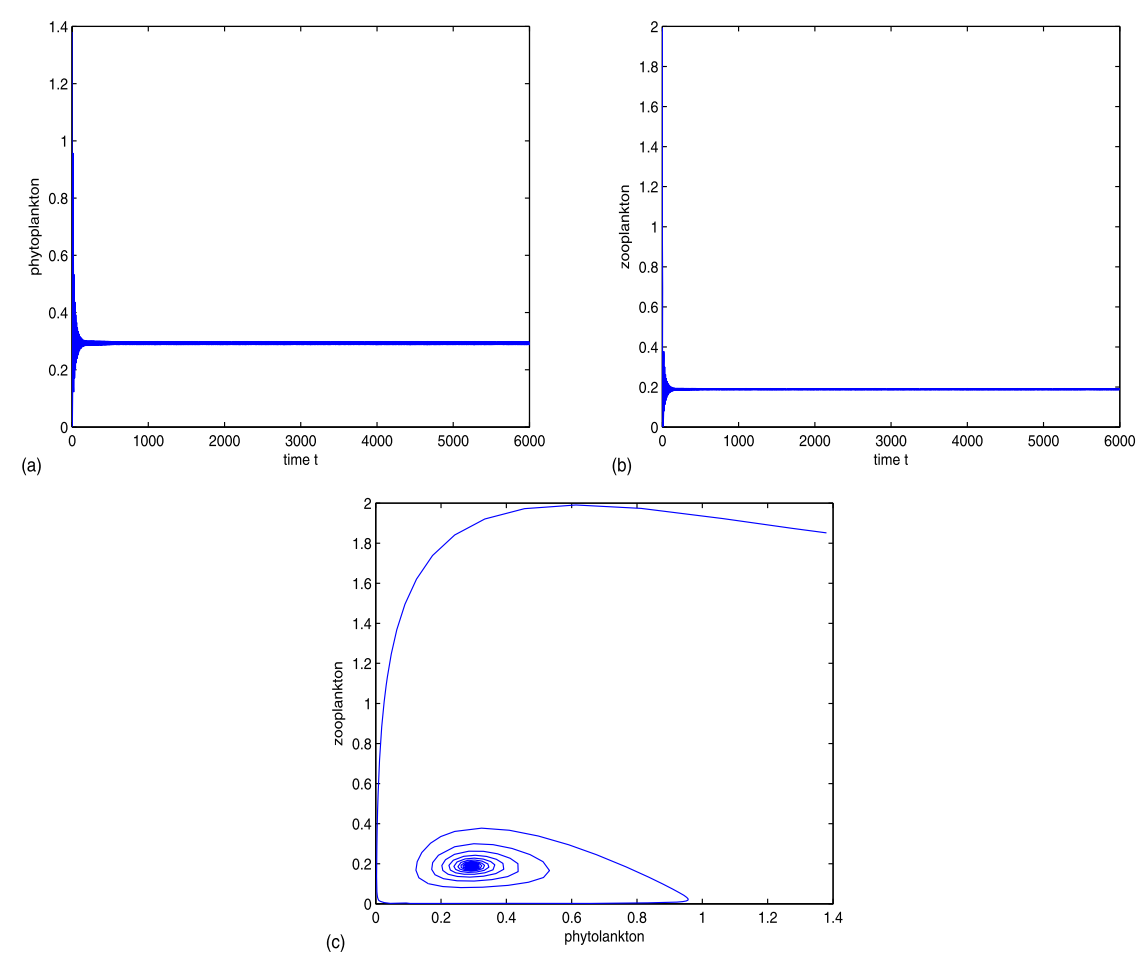

Figure 5 The local stability of the solution of system (4). (a)-(b) Time series. (c) Phase portrait. We take the parameters $a=1, b=8, c=3.09, d=1.2, e=0.0005, h=1.1$, and $g=8$. Numerical simulations show that for system (4) the positive equilibrium point $E^{*}$ is locally asymptotically stable for $\tau=0.11<\hat{\tau}=0.4422694125$.

in Figure 6. The model simulation also shows that the delay can enhance the amplitude of the periodic solution when the periodic solutions exist, which means that the delay has a large effect on the dynamics of system (4), as proved in Figure 7. In additional, from Section 3.5 , we can easily obtain $c_{1}(0)=-837.7866+8777.8357 i, \mu_{2}=12147.066>0$, $\beta_{2}=-1675.5732<0$, and $T_{2}=-51246.7726<0$, which shows that the Hopf bifurcation is supercritical and the bifurcation periodic solutions are stable with decreasing period. 

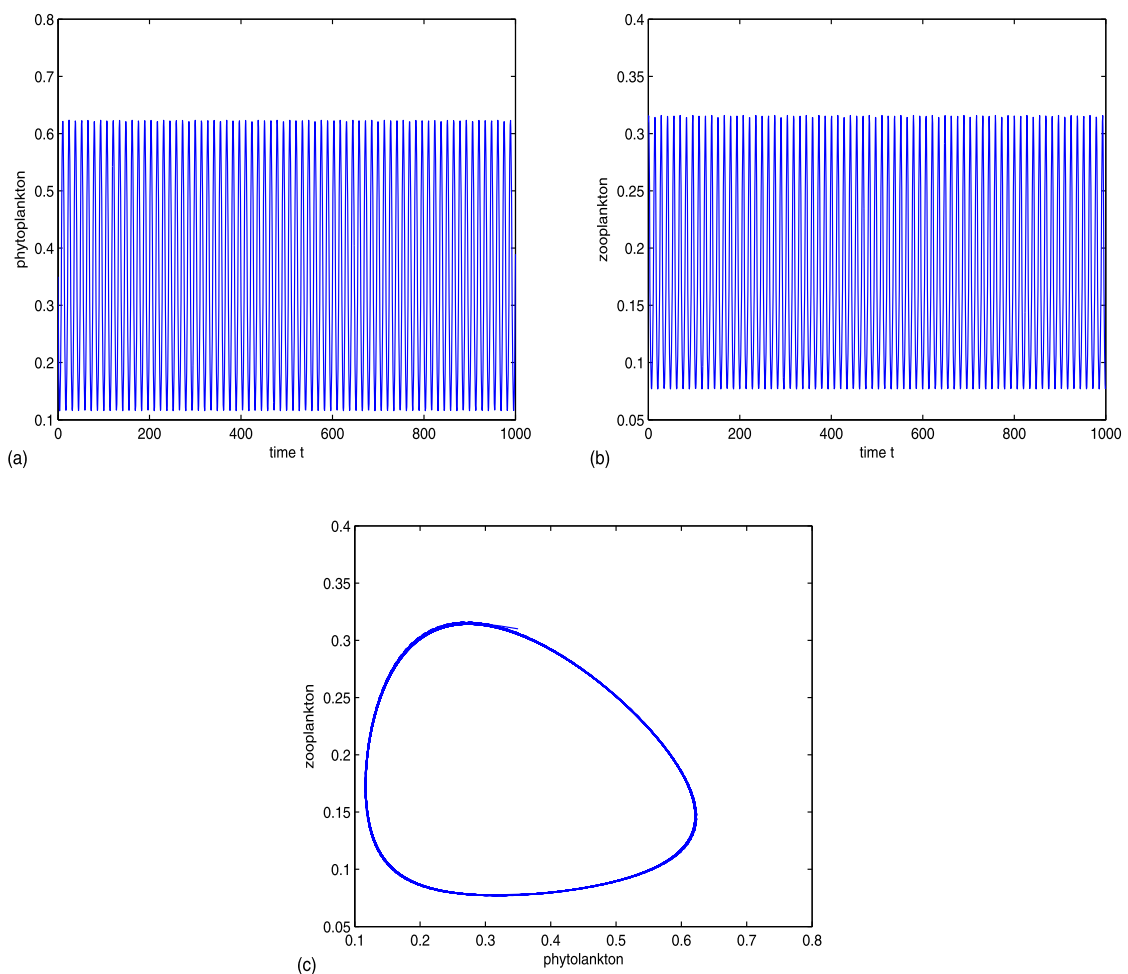

Figure 6 The stability of periodic solutions of system (4). (a)-(b) Time series. (c) Phase portrait. The parameters are the same as given in Figure 5. Numerical simulation shows that for system (4) the positive equilibrium point $E^{*}$ is unstable for $\tau=0.59>\hat{\tau}=0.4422694125$ and the periodic phenomenon caused by a Hopf bifurcation occurs. There exists a positive period-1 solution of system (4).
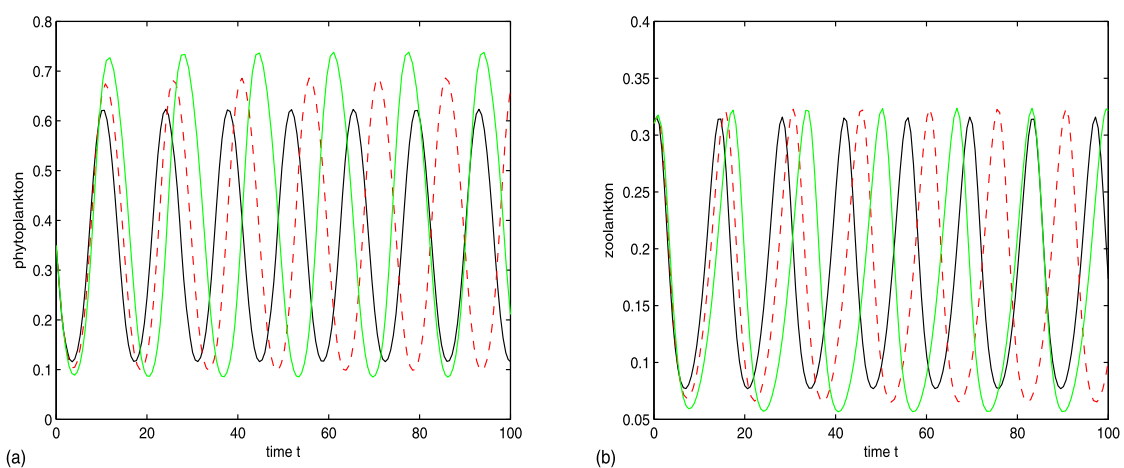

Figure 7 The effects of different time delays on the existence of periodic solutions. We take the same parameters as in Figure 5. The black line is for $\tau=0.59$, the red dashed line for $\tau=0.75$, and the green line for $\tau=0.95$. (a) The time series of the phytoplankton. (b) The time series of the zooplankton.

\section{Discussion and conclusion}

Over the two decades, a great deal of research has been devoted to the dynamics of the plankton ecosystem, however, a clear understanding of the mechanisms that cause the plankton blooms is still lacking and, therefore, it has been remained an interesting area of research for many ecologists and mathematical biologists [30]. In this paper, an attempt has been made to study the dynamic behaviors of a phytoplankton-zooplankton system 
with a Crowley-Martin functional response and its corresponding delayed version. In order to see how the length of gestation delay affects the dynamical behavior, we have first analyzed the system without delay and obtained the parameters conditions for the permanence (Theorem 2.10). That is, if $\left(\mathrm{H}_{1}\right)$ and $\left(\mathrm{H}_{2}\right)$ hold, the phytoplankton and zooplankton populations of the system will have lasting coexistence. It is also derived that the system in the absence of delay remains locally asymptotically stable when $b>d$ and $a b>c b-c d+e$ (Theorem 2.13), which means that phytoplankton blooms will not occur. More interesting, it can be concluded that the positive interior equilibrium of the system without delay is globally asymptotically stable under the conditions of Theorem 2.14 (see Figure 1). From the biological point of view, it leads the phytoplankton and zooplankton populations to coexist.

Next we have presented analogous results for the delayed system and considered the stability and direction of the Hopf bifurcation. It is should be noted that the system in the presence of delay is also globally asymptotically stable if $\left(\mathrm{H}_{1}\right)-\left(\mathrm{H}_{2}\right), a>b Z^{*}\left(c+e Q_{1}\right)$, and (i)-(ii) hold (see Figure 2). In the work of $[2,12,34,35,42,48]$, one showed that the time delay can cause a stable equilibrium to become unstable and even a switching of stabilities in their system, in other words, a time delay which incorporates in a biological ecosystem can lead to the ecosystem's steady state switch from stable to unstable. In particular, in [34], the authors also investigated the dynamical behavior of a phytoplankton-zooplankton system with a gestation delay, they observed that the gestation delay has a destabilizing effect on the system dynamics. However, in the present paper, based on the comparative analysis of the global stability of the numerical simulation results, it was found that the delay (gestation) did not change the stability of the system (Figure 1(a) and (b) and Figure 2(a) and (b), Figures 3 and 4). In [4], where the global stability and the Hopf bifurcation in a zooplankton-phytoplankton system was studied, the obtained results also showed that the time delay did not change the stability of the system. Additionally, our results also indicated that the delay can accelerate the process of its stability (Figures 1(c) and 2(c)). Although the delay cannot change the stability of the system, it should be emphasized that the delays were bound to influence the process. These results may be very meaningful to study the dynamics of phytoplankton-zooplankton interaction and may have great importance for research on plankton bloom.

In addition, it has also been shown that the time delay can induce instability and oscillations via a Hopf bifurcation in the system in the case of the presence of delay, and thereafter, switching of stability occurs. In other words, the stability of system (4) can be changed by the delay. More specifically, we have established that when $\tau<\hat{\tau}=$ 0.4422694125 , the positive interior equilibrium $E^{*}$ is stable under certain parametric restrictions mentioned in Theorem 3.5. However, when the time delay $\tau$ exceeds the threshold value $\hat{\tau}=0.4422694125$, the delayed phytoplankton-zooplankton system will undergo a Hopf bifurcation and exhibit a periodic orbit around the coexisting equilibrium point $E^{*}$ (see Figure 6). It should be noted that gestation delay can enhance the population fluctuations when the delay is long enough, however, our analytical results demonstrated that population fluctuations will not appear if $h+j_{12} \frac{g}{b}>j_{11}, j_{12}\left(j_{21}+\frac{g}{b} j_{11}\right)+h j_{11}<0$ and any of $\left(\mathrm{s}_{1}\right)$, $\left(\mathrm{s}_{2}\right),\left(\mathrm{s}_{3}\right)$ hold (Theorem 3.5). From the biological point of view, it implies the disappearance of bloom of plankton populations. Consequently, the gestation delay on CrowleyMartin functional response of the zooplankton can ensure some mechanism for controlling the plankton bloom. This may be helpful to study the problem of plankton bloom. 
Furthermore, by virtue of the normal form method and center manifold theory, we have derived that a Hopf bifurcation is supercritical $\left(\mu_{2}>0\right)$ and the bifurcation periodic solutions are stable with decreasing period $\left(\beta_{2}<0\right.$ and $\left.T_{2}<0\right)$.

Finally, although the study of the problems of the limit in theory study, the data of numerical simulations are not based on real world survey, it can be seen that numerical simulation results support our analytical findings. Hence, our work may be helpful to field investigation and experimental research in the real situation, as well as may also be helpful for qualitative research into similar real systems in nature. Nevertheless, in the real world, the environment of the planktonic creature is random, we also believe that our theoretical results will be useful in the study of delayed Crowley-Martin-type phytoplanktonzooplankton model systems in a stochastic environment, which we leave for future work.

Competing interests

The authors declare that they have no competing interests.

\section{Authors' contributions}

All authors contributed equally and significantly in writing this article. All authors read and approved the final manuscript.

\section{Author details}

${ }^{1}$ School of Mathematics and Information Science, Wenzhou University, Wenzhou, Zhejiang 325035, China. ${ }^{2}$ School of Life and Environmental Science, Wenzhou University, Wenzhou, Zhejiang 325035, China. ${ }^{3}$ Zhejiang Provincial Key Laboratory for Water Environment and Marine Biological Resources Protection, Wenzhou University, Wenzhou, Zhejiang 325035, China.

\section{Acknowledgements}

This work was supported by the National Natural Science Foundation of China (Grant Nos. 31370381 and 31570364).

Received: 15 June 2016 Accepted: 6 December 2016 Published online: 04 January 2017

\section{References}

1. Reigada, R: Plankton blooms induced by turbulent flows. Philos. Trans. R. Soc. Lond. B, Biol. Sci. 270, 875-880 (2003)

2. Saha, T, Bandyopadhyay, M: Dynamical analysis of toxin producing phytoplankton-zooplankton interactions. Nonlinear Anal., Real World Appl. 10, 314-332 (2009)

3. Huppert, A, Blasius, B, Stone, L: A model of phytoplankton blooms. Am. Nat. 159, 156-171 (2002)

4. LV, YF, Cao, JZ, Song, J, Yuan, R, Pei, YZ: Global stability and Hopf-bifurcation in a zooplankton-phytoplankton model. Nonlinear Dyn. 76, 345-366 (2014)

5. Chaudhuri, S, Roy, S, Chattopadhyay, J: Phytoplankton-zooplankton dynamics in the 'presence' or 'absence' of toxic phytoplankton. Appl. Math. Comput. 225, 102-116 (2013)

6. Fan, AJ, Han, P, Wang, KF: Global dynamics of a nutrient-plankton system in the water ecosystem. Appl. Math. Comput. 219, 8269-8276 (2013)

7. Zhang, TR, Wang, WD: Hopf bifurcation and bistability of a nutrient-phytoplankton-zooplankton model. Appl. Math. Model. 36, 6225-6235 (2012)

8. Luo, JH: Phytoplankton-zooplankton dynamics in periodic environments taking into account eutrophication. Math. Biosci. 245, 126-136 (2013)

9. Dai, CJ, Zhao, M, Yu, HG, Wang, YP: Delay-induced instability in a nutrient-phytoplankton system with flow. Phys. Rev. E 91, 032929 (2015)

10. Mei, DB, Zhao, M, Yu, HG, Dai, CJ, Wang, Y: Nonlinear dynamics of a nutrient-phytoplankton model with time delay. Discrete Dyn. Nat. Soc. 2015, Article ID 939187 (2015)

11. Deng, YL, Zhao, M, Yu, HG, Wang, Y: Dynamical analysis of a nitrogen-phosphorus-phytoplankton model. Discrete Dyn. Nat. Soc. 2015, Article ID 823026 (2015)

12. Abbas, S, Banerjee, M, Hungerbühler, N: Existence, uniqueness and stability analysis of allelopathic stimulatory phytoplankton model. J. Math. Anal. Appl. 367, 249-259 (2010)

13. Chakraborty, K, Das, K: Modeling and analysis of a two-zooplankton one-phytoplankton system in the presence of toxicity. Appl. Math. Model. 39, 1241-1265 (2015)

14. Kartal, S, Kar, M, Kartal, N, Gurcan, F: Modelling and analysis of a phytoplankton-zooplankton system with continuous and discrete time. Math. Comput. Model. Dyn. Syst. 22, 539-554 (2016)

15. Rhee, GY, Gotham, IJ: The effect of environmental factors on phytoplankton growth: temperature and the interactions of temperature with nutrient limitation. Limnol. Oceanogr. 26, 635-648 (1981)

16. Stomp, M, Huisman, J, de Jongh, F, Veraart, AJ, Gerla, D, Rijkeboer, M, Ibelings, BW, Wollenzien, UI, Stal, LJ: Adaptive divergence in pigment composition promotes phytoplankton biodiversity. Nature 432, 104-107 (2004)

17. Sklaski, GT, Gilliam, JF: Functional response with predator interference: viable alternative to Holling type II model. Ecology 82, 3083-3092 (2001)

18. Crowley, PH, Martin, EK: Functional response and interference within and between year classes of a dragonfly population. J. North Am. Benthol. Soc. 8, 211-221 (1989)

19. Freedman, HI: Deterministic Mathematical Models in Population Ecology. Dekker, New York (1980) 
20. Seifert, G: Asymptotical behavior in a three-component food chain model. Nonlinear Anal. 32, 749-753 (1998)

21. Fan, A, Han, P, Wang, K: Global dynamics of a nutrient-plankton system in the water ecosystem. Appl. Math. Comput. 219, 8269-8276 (2013)

22. Moroz, I, Cropp, R, Norbury, J: Chaos in plankton models: foraging strategy and seasonal forcing. Ecol. Model. 332 103-111 (2016)

23. Truscott, JE, Brindley, J: Ocean plankton populations as excitable media. Bull. Math. Biol. 56, 981-998 (1994)

24. Yu, HG, Zhao, M, Agarwal, RP: Stability and dynamics analysis of time delayed eutrophication ecological model based upon the Zeya reservoir. Math. Comput. Simul. 97, 53-67 (2014)

25. Chakraborty, K, Chakraborty, M, Kar, TK: Bifurcation and control of a bioeconomic model of a prey-predator system with a time delay. Nonlinear Anal. Hybrid Syst. 5, 613-625 (2011)

26. Li, WS, Chang, YK, Nieto, JJ: Solvability of impulsive neutral evolution differential inclusions with state-dependent delay. Math. Comput. Model. 49, 1920-1927 (2009)

27. Li, XD, Fu, XL: Effect of leakage time-varying delay on stability of nonlinear differential systems. J. Franklin Inst. 350 $1335-1344$ (2013)

28. Liu, SQ, Beretta, E, Breda, D: Predator-prey model of Beddington-DeAngelis type with maturation and gestation delays. Nonlinear Anal., Real World Appl. 11, $4072-4091$ (2010)

29. Dai, CJ, Zhao, M, Yu, HG: Dynamics induced by delay in a nutrient-phytoplankton model with diffusion. Ecol. Complex. 26, 29-36 (2016)

30. Sharma, A, Sharma, AK, Agnihotri, K: Analysis of a toxin producing phytoplankton-zooplankton interaction with Holling IV type scheme and time delay. Nonlinear Dyn. 81, 13-25 (2015)

31. Sharma, A, Sharma, AK, Agnihotri, K: The dynamic of plankton-nutrient interaction with delay. Appl. Math. Comput. 231, 503-515 (2014)

32. Wang, Y, Wang, HB, Jiang, WH: Hopf-transcritical bifurcation in toxic phytoplankton-zooplankton model with delay. J. Math. Anal. Appl. 415, 574-594 (2014)

33. Zhao, HY, Huang, XX, Zhang, XB: Hopf bifurcation and harvesting control of a bioeconomic plankton model with delay and diffusion terms. Physica A 421, 300-315 (2015)

34. Rehim, M, Imran, M: Dynamical analysis of a delay model of phytoplankton-zooplankton interaction. Appl. Math. Model. 36, 638-647 (2012)

35. Wang, Y, Jiang, W Wang, H: Stability and global Hopf bifurcation in toxic phytoplankton-zooplankton model with delay and selective harvesting. Nonlinear Dyn. 73, 881-896 (2013)

36. Gakkhar, S, Singh, A: A delay model for viral infection in toxin producing phytoplankton and zooplankton system. Commun. Nonlinear Sci. Numer. Simul. 15, 3607-3620 (2010)

37. Brikhoff, G, Rota, GC: Ordinary Differential Equations. Wiley, New York (1982)

38. Tripathi, JP, Tyagi, S, Abbas, S: Global analysis of a delayed density dependent predator-prey model with Crowley-Martin functional response. Commun. Nonlinear Sci. Numer. Simul. 30, 45-69 (2015)

39. Chen, FD, Li, Z, Chen, X, Jitka, L: Dynamic behaviours of a delay differential equation model of plankton allelopathy. J. Comput. Appl. Math. 206, 733-754 (2007)

40. Song, YL, Han, MA, Wei, JJ: Stability and Hopf bifurcation analysis on simplified BAM neural network with delays. Physica D 200, 185-204 (2005)

41. Gopalsamy, K: Stability and Oscillations in Delay Differential Equations of Population Dynamics. Kluwer Academic, Dordrecht (1992)

42. Das, K, Ray, S: Effect of delay on nutrient cycling in phytoplankton-zooplankton interactions in estuarine system. Ecol. Model. 215, 69-76 (2008)

43. Freedman, HI: Models of competition in the chemostat with instantaneous and delayed nutrient recycling. J. Math. Biol. 31, 513-527 (1993)

44. Wang, WM, Zhu, YN, Cai, YL, Wang, WJ: Dynamical complexity induced by Allee effect in a predator-prey model. Nonlinear Anal., Real World Appl. 16, 103-119(2014)

45. Freedman, HI, Rao, VSH: The trade-off between mutual interference and time lags in predator-prey systems. Bull. Math. Biol. 45, 991-1004 (1983)

46. Hassard, B, Kazarinoff, D, Wan, Y: Theory and Application of Hopf Bifurcation. Cambridge University Press, Cambridge (1981)

47. Hale, JK, Verduyn, SM: Introduction to Functional Differential Equations. Springer, New York (1993)

48. Sharma, AK, Sharma, A, Agnihotri, K: Bifurcation behaviors analysis of a plankton model with multiple delays. Int. J. Biomath. 9, $1650086(2016)$

\section{Submit your manuscript to a SpringerOpen ${ }^{\ominus}$ journal and benefit from:}

- Convenient online submission

- Rigorous peer review

- Immediate publication on acceptance

- Open access: articles freely available online

- High visibility within the field

- Retaining the copyright to your article

Submit your next manuscript at $>$ springeropen.com 